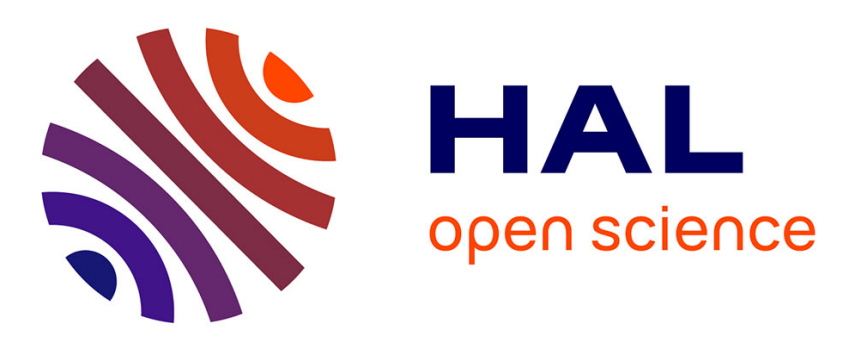

\title{
Synthesis of N-(Hetero)arylconvolvine Derivatives through a Palladium-Catalyzed Buchwald-Hartwig Cross-Coupling
}

Manel Hassine, Hichem Ben Jannet, Noureddine Ghermani, Mouad Alami, Samir Messaoudi

\section{To cite this version:}

Manel Hassine, Hichem Ben Jannet, Noureddine Ghermani, Mouad Alami, Samir Messaoudi. Synthesis of N-(Hetero)arylconvolvine Derivatives through a Palladium-Catalyzed Buchwald-Hartwig CrossCoupling. Synthesis: Journal of Synthetic Organic Chemistry, 2019, 10.1055/s-0039-1690238 . hal02396680

\section{HAL Id: hal-02396680 \\ https://hal.science/hal-02396680}

Submitted on 6 Dec 2019

HAL is a multi-disciplinary open access archive for the deposit and dissemination of scientific research documents, whether they are published or not. The documents may come from teaching and research institutions in France or abroad, or from public or private research centers.
L'archive ouverte pluridisciplinaire HAL, est destinée au dépôt et à la diffusion de documents scientifiques de niveau recherche, publiés ou non, émanant des établissements d'enseignement et de recherche français ou étrangers, des laboratoires publics ou privés. 


\title{
Synthesis of $\mathrm{N}$-(hetero)aryl- \\ Convolvine Derivatives Through a \\ Palladium-Catalyzed Buchwald- \\ Hartwig Cross-Coupling
}

\author{
Manel Hassine ${ }^{a, b}$ \\ Hichem Ben Jannet*b \\ NourEddine, Ghermani ${ }^{\mathrm{C}}$ \\ Mouad Alami ${ }^{a}$ \\ Samir Messaoudi*a \\ ${ }^{a}$ BioCIS-UMR 8076, Univ. Paris-Sud, CNRS, University Paris- \\ Saclay, Châtenay-Malabry, France \\ b University of Monastir, Faculty of Science of Monastir, \\ Laboratory of Heterocyclic Chemistry, Natural Products and \\ Reactivity (LR11ES39), Team: Medicinal Chemistry and Natural \\ Products, Avenue of Environment, 5019 Monastir, Tunisia. \\ 'Institut Galien UMR 8612, Univ. Paris-Sud, CNRS, University \\ Paris-Saclay, Châtenay-Malabry, France
}

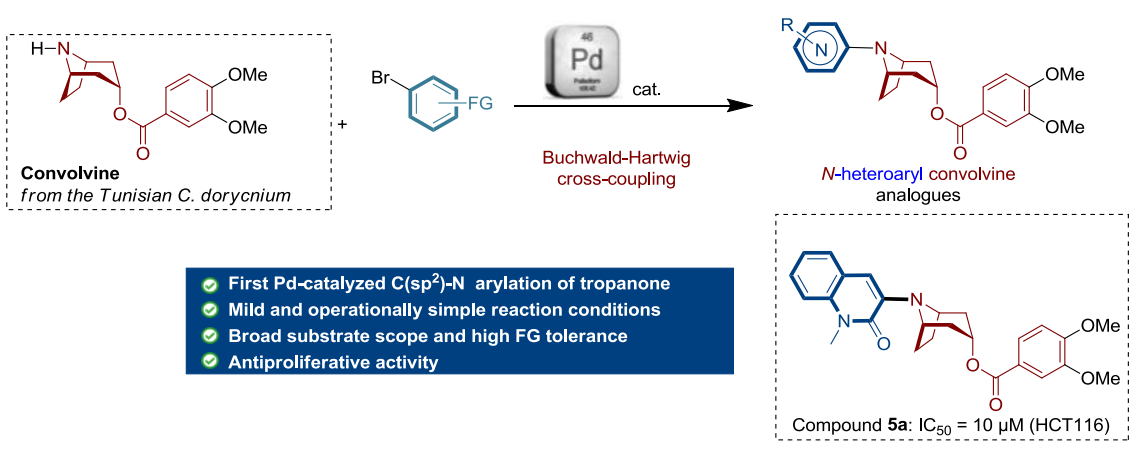

*samir.messaoudi@u-psud.fr, hichem.bjannet@gmail.com

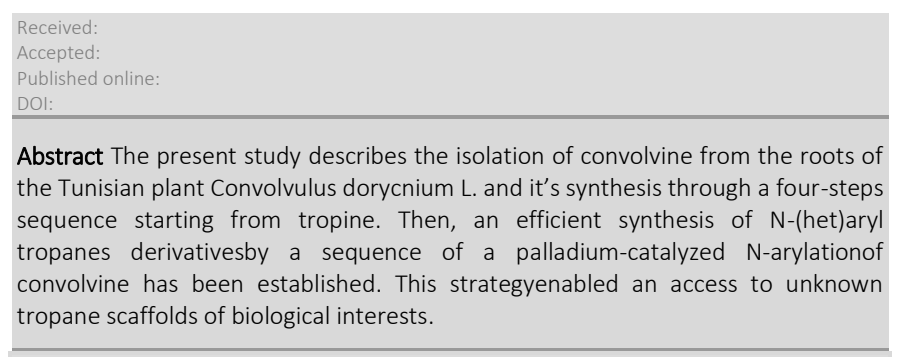

Key words convolvine, Buchwald-Hartwig reaction, Palladium catalysis, tropane

Tropane is an important nitrogen bicyclic motif found in many natural and synthetic compounds. ${ }^{1}$ Tropane derivatives are among the economically most important pharmaceuticals. ${ }^{2}$ More than twenty active pharmaceutical ingredients (APIs) containing the tropane moiety are manufactured by pharmaceutical industries as antispasmodics, anesthetics, antiemetrics and bronchodilators. ${ }^{3}$
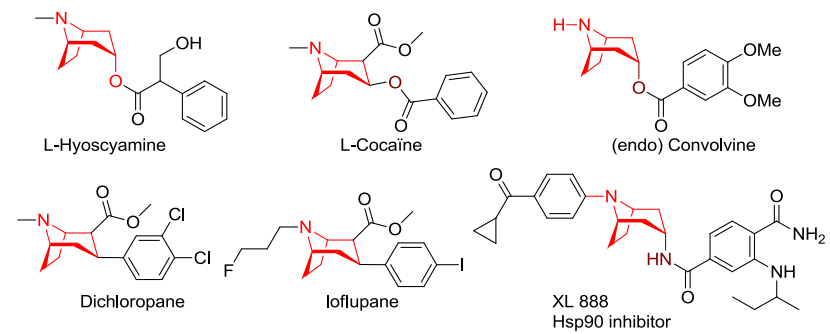

Figure 1. Tropane natural products and some semi-synthetic analogues.
The biological importance of some Convolvulus species and the high biological activity of the tropane alkaloids encouraged us to undertake a phytochemical study of Convolvulus dorycnium L. whose $n$-BuOH extract of the roots affording an interesting tropane natural compound Convolvine (Figure 1) that was isolated for the first time from this plant.

One of the interesting tropane-compound currently under clinical investigation is the tropanone-based inhibitor XL888 which is reported as a potent and selective ATP-competitive inhibitor of HSP90. ${ }^{4}$ In preclinical studies, XL888 inhibits the proliferation of a broad panel of human tumor cell lines and induces marked degradation of HSP90 client proteins. In addition, XL888 is highly active in multiple human tumor xenograft models in mice.

In the context of the development of news Hsp90 inhibitors, we reported in a previous study, that the 3-amidoquinolinone compound $6 \mathrm{BrCaQ}$ (Scheme 1) ${ }^{5},{ }^{6}$ is a highly potent HSP90 inhibitors. This derivative displayed antiproliferative activities ranging from 2 to $8 \mu \mathrm{M}$ against various cancer cell lines (MCF7, MDA MB231, Caco2, IGROV-1 and ISHIKAWA). Morever, this lead compound is able to induce a significant down regulation of several hsp90 client proteins (HER2, Raf-1 and cdk-4).

Because of the exciting activities of XL888 and 6BrCaQ, we were interesting whether the combination of the tropane core of XL888 and the quinoleine nucleus of $6 \mathrm{BrCaQ}$ in a one molecule may lead to more efficacious antiproliferative compounds. Herein, we planned to prepare a new series of $\mathrm{N}$-(hetero)aryl convolvine analogues (Figure 2), in which various chemical modifications at the heteroaromatic nucleus were performed in the aim to better understand the SAR in this novel series. In this article the synthesis and the biological evaluation of novel (hetero)aryl convolvine analogues 1-10 are described. Preliminary in vitro efficacy of these compounds in terms of antiproliferative activity is reported. 


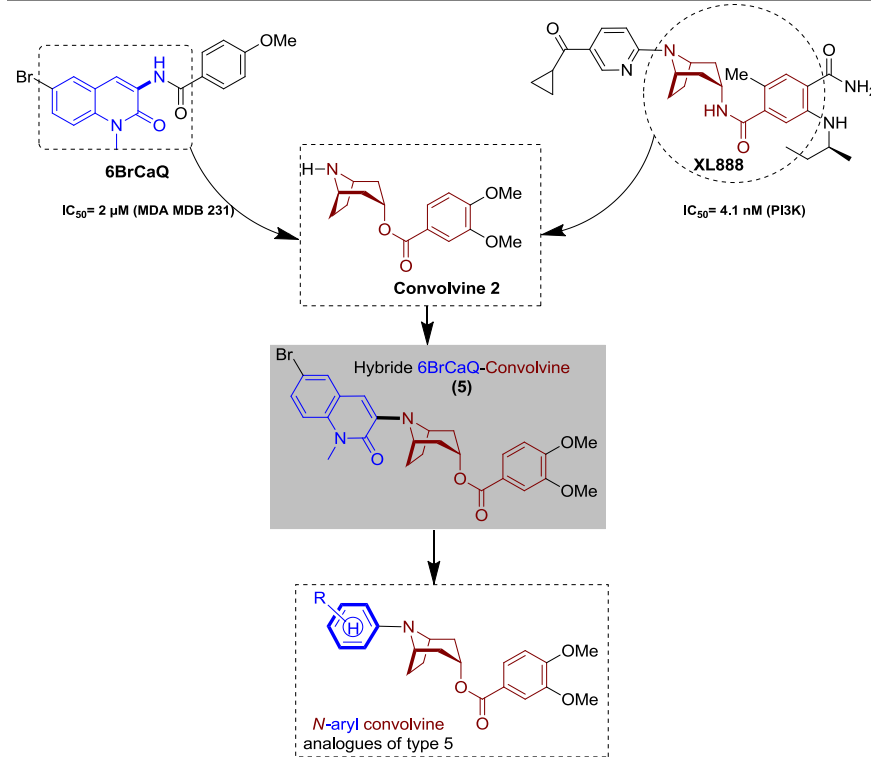

Scheme1. General strategy to N-(hetero)aryl convolvine analogues

Convolvine (Figure 1) was isolated as a white solid from the $n$-BuOH extract from the roots of $C$. from the Tunisian $C$. dorycnium. Its structure was evidenced through the ${ }^{1} \mathrm{H}$ and ${ }^{13} \mathrm{C}$ spectroscopy examination and by comparison with literature data. ${ }^{7}$ We note that this same compound has been also isolated from Convolvulus subhirsutus $^{8}$ and Convolvulus krauseanus ${ }^{9}$ without specifying the corresponding stereochemistry.

To have at our disposal a sufficient quantity of convolvine as a starting material for the further modifications, we envisioned to perform its synthesis in a gram scale rather than its isolation from plant. Accordingly, the synthesis commenced from $\mathrm{O}$-acylation of tropine with the pre-formed 2,3-dimethoxybenzoyl chloride. Our first attempt was performed according to the protocol of Maksay et $a^{10}{ }^{10}$ using $\mathrm{NEt}_{3}$ as a base in refluxing toluene. Under these conditions, the desired $\mathrm{N}$-methylconvolvine $\mathbf{3}$ was isolated in yield never exceed $20 \%$. This result is in concordance with the observation of Maksay et al. ${ }^{10}$ who's suggested that the acylation of tropine is usually difficult because of the sterically hindered hydroxyl group. To increase the yield of the $O$-acylation various reaction parameters were explored. Thus, when a solution of the tropine and the freshly pre-formed 2,3-dimethoxybenzoyl chloride in dichloromethane was stirred at room temperature overnight in the presence of a trimethylamine as a base and a catalytic amount of DMAP, the $N$-methylconvolvine $\mathbf{3}$ was isolated after purification in good $64 \%$ yield (Scheme 2). It is important to note that the acylation reaction was also performed in a gram scale $(84 \mathrm{mmol}, 12$ g), and the product 3 was isolated in a similar yield (66\%).

The X-ray crystal structure of $\mathrm{N}$-methylconvolvine $3^{11}$ depicted in scheme 2 shows that the conformation of the tropane scaffold and the aryl nucleus are not planar with an dihedral angle around $90^{\circ}$. Moreover, the endo-stereochemistry of the tropine nucleus which is corroborated with the NMR data, was conserved during the acylation process as confirmed by the X-crystal structure analysis of compound 3 (Scheme 2).

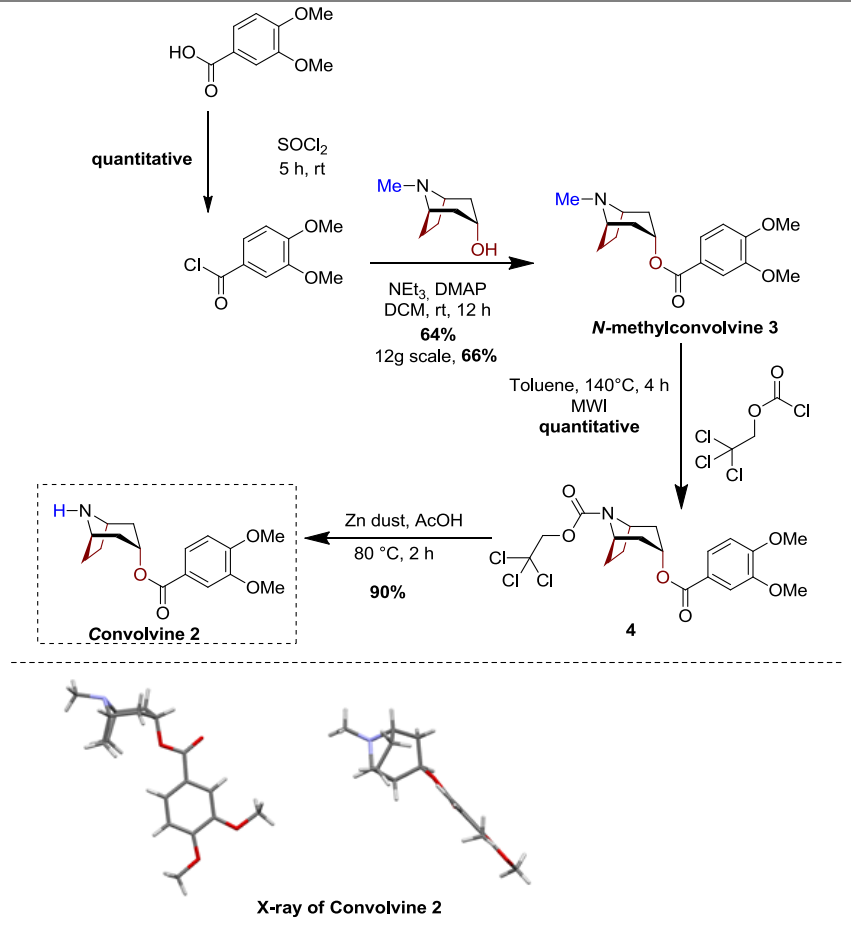

Scheme 2. Synthesis of convolvine 2 .

With the $N$-methylconvolvine in our hand, we next turned our attention to the deprotection of the nitrogen atom in the intermediate 3 . The challenge of this transformation is to achieve the demethylation while keeping intact the ester function. Several methods are known for the demethylation of tropane derivatives ${ }^{12}$ such the use of ethyl chloroformate or 2,2,2-trichloroethyl chloroformate as demethylating agents. These methods proceed through two concomitant steps; (i) acylation/demethylation of the nitrogen and (ii) deacylation of the carbamate function. All our attempts to synthesis the endo-convolvine $\mathbf{2}$ using these two demethylating reagents, under several reported conditions failed. At this stage, we decided to perform step by step the protocol of the demetylation when 2,2,2-trichloroethyl chloroformate was used. In the first step, the carbamate 4 resulting from acylation of the $\mathrm{N}$ methylconvolvine under microwave conditions followed by $\mathrm{N}$ demethylation of the ammonium intermediate, was obtained in a quantitative yield (Scheme 2). Removal of the trichloroethoxycarbonyl group was then achieved using zinc dust in acetic acid solution. Under these conditions the endo-convolvine 2 was isolated in a $90 \%$ yield as a pure endo-conformer without any affecting the ester group. This sequence allows us obtaining the convolvine $\mathbf{2}$ in $56 \%$ overall yield even when achieving the synthesis in a gram scale $(2 \mathrm{~g})$.

Upon completion of the synthesis of the convolvine, we turned our attention to functionalization of the nitrogen atom of convolvine through Pd-catalyzed cross coupling. The main issue of this coupling is to maintain the benzoic ester group intact during the cross coupling under basic conditions. To this end, we examined the coupling of 3-bromoquinoline $\mathbf{1 a}$ with convolvine $\mathbf{2}$ as a model study under various source of palladium/ligand catalysts, bases and solvents. Representative results from this study are summarized in The reaction of 1a (1 equiv) with 2 ( 2 equiv) was first investigated under our previously reported procedure $\left[\mathrm{Pd}(\mathrm{OAc})_{2}\right.$. (5mol\%), XantPhos (5 mol\%), $\mathrm{Cs}_{2} \mathrm{CO}_{3}$, dioxane, at $\left.110{ }^{\circ} \mathrm{C}\right]^{[13]}$ (Table 1, entry 
1). Unfortunately, this coupling reaction failed and only the starting materials were recovered unchanged. Using the monodentate ligand XPhos instead the bidentate XantPhos was also unsuccessful (entry 2). Interestingly, switching from $\mathrm{Cs}_{2} \mathrm{CO}_{3}$ as the base to the more basic $t$-BuONa, the coupling reaction furnished $\mathbf{5 a}$ in a $12 \%$ yield(entry 3 ). This first result indicates clearly that the reactivity of the amine of convolvine is far to be trivial. To increase the yield of $\mathbf{5 a}$, we examined the influence of other reaction parameters. Pleasantly, when the coupling reaction was performed by using the ratio of $\mathrm{Pd} / \mathrm{L}=1: 2\left(5 \mathrm{~mol} \% \mathrm{Pd}(\mathrm{OAc})_{2}\right.$ and $10 \mathrm{~mol} \%$ of XPhos), the yield was improved up to $66 \%$ and the benzoic ester was maintained intact (entry 5). By increasing the amount of the base (from 1.5 equiv to 2 equiv), we managed to isolate $95 \%$ of the desired product $\mathbf{5 a}$ (entry 6).

Table 1. Survey of reaction conditions for the $N$-arylation of convolvine $\mathbf{2}$ with 3-bromoquinolinone $\mathbf{1 a}^{\mathrm{a}}$.

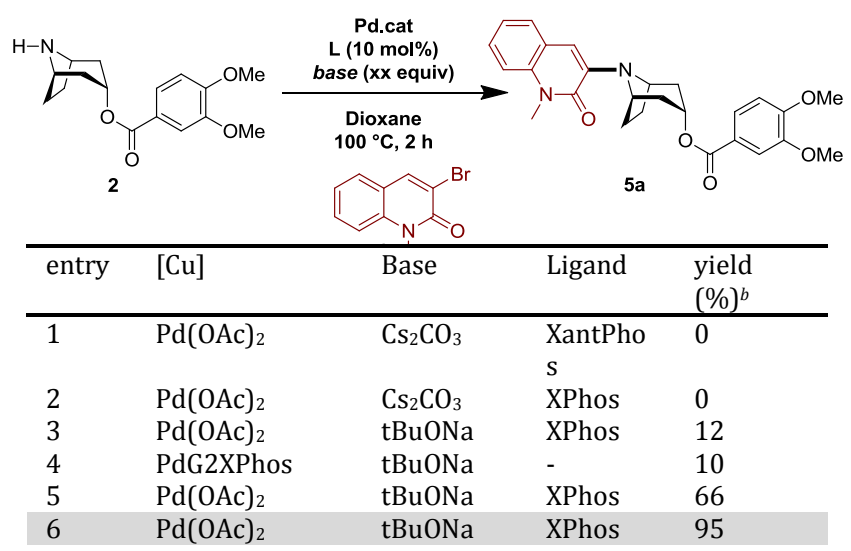

a3-bromoquinolinone 1a (1 equiv), convolvine2 (2 equiv), [ $\left.\mathrm{Pd}(\mathrm{OAc})_{2}\right]$ (5 mol \%), Ligand ( $5 \mathrm{~mol} \%$ ), base (1.5 equiv), $2 \mathrm{~h}, 100,[0.5 \mathrm{M}] .{ }^{b}$ Yield of isolated 5a. ${ }^{c}$

Motivated by these results, we next explored the scope of the coupling reaction of convolvine2 with various (hetero)aryl bromides. Gratifyingly, all the $\mathrm{N}$-arylations proceeded cleanly to give the substituted aryl $\mathrm{N}$-convolvine analogues $5 \mathbf{a}$-oin good to excellentyields. As depicted in Table 2, convolvine $\mathbf{2}$ was readily coupled with aryl bromides having para and meta electronwithdrawing $\left(-\mathrm{F},-\mathrm{CF}_{3},-\mathrm{Cl},-\mathrm{NO}_{2},-\mathrm{NC}\right)$ or electron-donating $(-\mathrm{OMe})$ substituents to give aryl $N$-convolvine $5 b-f, 5 j, 6 l$ and $5 \mathrm{~m}$ in good yields.In addition, the sterically demanding ortho substitution pattern engaged in the coupling reaction of $\mathbf{2}$, furnishing compounds 5gand $\mathbf{5 j}$ having an ortho substituent group. Interestingly, heteroarene such as 3-bromoquinolinone, 5-bromoindole and 3bromopyridin were good substrates in this reaction (compounds $\mathbf{5 a}$, 5n-o). Of note, in the case of coupling with di-bromoquinolinone, only dicoupling product $\mathbf{6 0}$ was isolated as the single product in $43 \%$ yield.
Upon completion of their syntheses, the in vitro activity of convolvine derivatives 5a-o was evaluated by their growthinhibitory potency against a human colon carcinoma HCT-116 cells at the concentrations of $10^{-5} \mathrm{M}$. The quantification of cell survival in this cell line was established by using MTT assays after $72 \mathrm{~h}$ exposure (Table 2), and $\mathrm{GI}_{50}$ values were determined at the concentration required to produce $50 \%$ inhibition. Taxotere ${ }^{\circledR}$ was used as a positive control $\left(\mathrm{IC}_{50}=1 \mathrm{nM}\right)$.

The results of this study, summarized in Table 2, demonstrated that all tested compounds were able to decrease the cell viability in HCT116 cells until 19-79 survival compared to the reference compound $6 \mathrm{BrCaQ}(10 \%$ survival) indicating the biological potential of convolvine pharmacophore in this series. Interestingly, the combination of the quinolinone nucleus with convolvine moiety (5a) induced a significant decrease of the cell viability in HCT-116 cells (19\% survival) when compared with the reference $6 \mathrm{BrCaQ}(10 \%$ survival). Pleasantly, the growth inhibition value $\mathrm{GI}_{50}$ for compound 5a $\left(\mathrm{GI}_{50}=10 \mu \mathrm{M}\right)$ was measured and was found to be approximatively the same that the reference $6 \mathrm{BrCaQ}\left(\mathrm{GI}_{50}=8 \mu \mathrm{M}\right)$. This result clearly validates our initial strategy. Substitution of the nitrogen of the convolvine by an indole nucleus (compound $\mathbf{5 m}$ )did not produce compound that inhibit cell growth more effectively than the $\mathbf{5 a}$ (survival $=57 \%, \mathrm{GI}_{50}=63 \mu \mathrm{M}$ ). Another important observation is that the disubstituted convolvine $\mathbf{5 0}$ affects slightly the growth of HCT-116 cells (54\% survival) when compared with the monosubstituted convolvine 5a (19\% survival). These results clearly suggest that the presence of a bulky substituent at the C-7 position of the quinolinone nucleus affect cell viability. One can be noted that convolvine $\mathbf{2}$ with free-NH is less cytotoxic since $60 \%$ of cells survive when incubated with convolvine 2 .

In conclusion, we developed an efficient and practical protocol for the $\mathrm{N}$-arylation of the tropane nucleus of convolvine without affecting the benzoic ester part. This transformation exhibited broad substrate scope with respect to the aryl bromide partners. Through this methodology, we designed and synthesized a series of $\mathrm{N}$ (hetero)aryl-convolvines, and we identified, compound $\mathbf{5 a}$ displaying the most stronger antiproliferative activity against HCT116 cell lines. These preliminary results show the biological potential of convolvine pharmacophore and suggest that pharmacomodulations of this compound may increase its biological activity.

\section{Experimental section}

\section{General experimental methods:}

The compounds were all identified by usual physical methods, e.g., ${ }^{1} \mathrm{H}$ NMR, ${ }^{13} \mathrm{C}$ NMR, IR, MS (ESI). ${ }^{1} \mathrm{H}$ and ${ }^{13} \mathrm{C}$ NMR spectra were measured in $\mathrm{CDCl}_{3}$, DMSO- $d_{6}$ with a Bruker Avance-300. ${ }^{1} \mathrm{H}$ chemical shifts are reported in ppm from an internal standard TMS or of residual solvent peak. ${ }^{13} \mathrm{C}$ chemical shifts are reported in ppm from the residual solvent peak. IR spectra were measured on a Bruker Vector 22 
Table 2. Scope of the coupling reaction of convolvine 2 with various (hetero)aryl bromides and cytotoxic activity against HCT-116 cells.
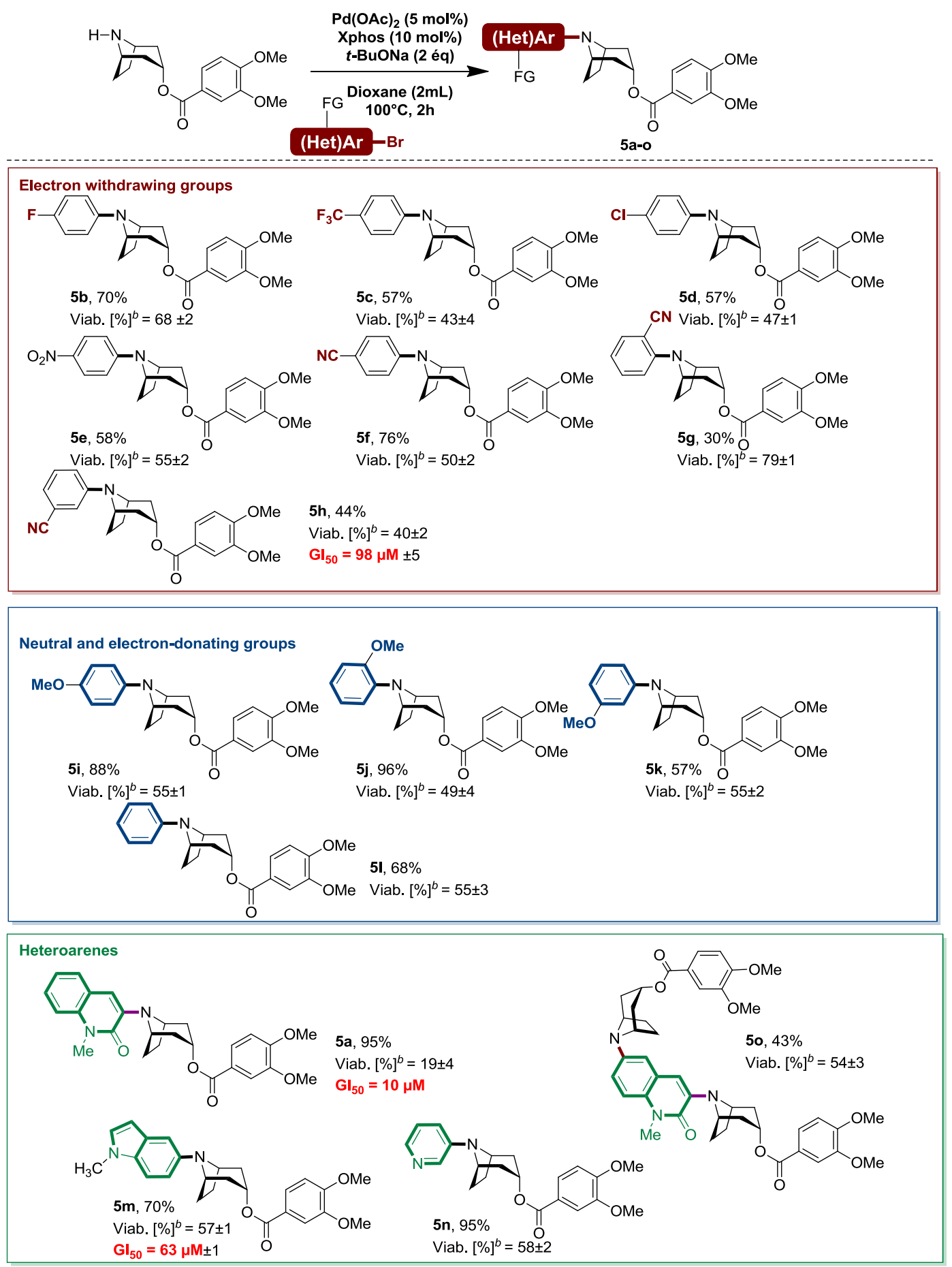

a. Reaction conditions: Convolvine (1 equiv), aryl bromide (2 equiv), Pd(OAc) 2 (5 mol\%), Xphos (10 mol\%), $t$-BuONa (2 equiv), Dioxane at $100^{\circ} \mathrm{C}, 2 \mathrm{~h} .{ }^{b}$ Value of the anti-proliferative effect (\% of viable cells compared to untreated cells $100 \%$ ) of convolvine analoguesin HCT-116 cell line at a concentration of $10^{-5} \mathrm{M}$ (MTT method). 
spectrophotometer. Analytical TLC was performed on Merck precoated silica gel $60 \mathrm{~F}$ plates. Merck silica gel $60(0.015-0.040 \mathrm{~mm})$ was used for column chromatography. Melting points were recorded on a Büchi B-450 apparatus and are uncorrected. High resolution mass spectra (HR-MS) were recorded on a Bruker MicroTOF spectrometer, using ESI with methanol as the carrier solvent. Nominal and exact $\mathrm{m} / \mathrm{z}$ values are reported in Daltons.

\section{Plant material}

The roots of Convolvulus dorycnium L. were collected from the region of Sidi Khelifa, Sousse (Tunisia) on June 2012. The plant was identified by Prof. Fethia Harzallah-Skhiri in the Laboratory of Bioressources: Integrative Biology and Valorization at Higher Institute of Biotechnology of Monastir (Tunisia). A voucher specimen (CD-12) was deposited in the herbarium of the above laboratory.

\section{Extraction and isolation:}

Dry roots of $C$. dorycnium $(1.473 \mathrm{~kg})$ were extracted twice with Methanol-Water mixture (7:3) for three days at room temperature. Then the aqueous solution obtained after evaporation of solvent under vacuum, was partitioned with $n$ - $\mathrm{BuOH}$ to yield $100 \mathrm{~g}(6.7 \%)$ of the correspond extract. The $n-\mathrm{BuOH}$ extract was subjected to RP-18 flash column chromatography with gradient elution $\left(\mathrm{H}_{2} \mathrm{O} ; \mathrm{H}_{2} \mathrm{O} / \mathrm{MeOH}, 90: 10\right.$ to 30:70), yielding twelve fractions $\left(\mathrm{F}_{1}-\mathrm{F}_{12}\right)$ of which the fraction 9 appears a pure convolvine( $300 \mathrm{mg})$ according to TLC analysis.

\section{General information:}

All reactions were conducted under an argon atmosphere. Solvents: cyclohexane, pentane, ethyl acetate (EtOAc), methylene chloride $\left(\mathrm{CH}_{2} \mathrm{Cl}_{2}\right)$ and methanol $(\mathrm{MeOH})$ for extraction and chromatography were of technical grade. The compounds were all identified by usual physical methods, i.e., 1D and 2D NMR (COSY, HSQC, HMBC and NOESY), IR, ESHRMS. ${ }^{1} \mathrm{H}$ and ${ }^{13} \mathrm{C}$ NMR spectra were measured in $\mathrm{CDCl}_{3}$ or Acetone $-d_{6}$ on a 300 and $400 \mathrm{MHz}$ spectrometer. ${ }^{1} \mathrm{H}$ chemical shifts are reported in parts per million from an internal standard TMS or of residual chloroform $(7.27 \mathrm{ppm})$. The following abbreviations are used: $\mathrm{m}$ (multiplet), s (singlet), br s (broad singlet), d (doublet), t (triplet) dd (doublet of doublet). ${ }^{13} \mathrm{C}$ chemical shifts are reported in parts per million from the central peak of deuteriochloroform (77.14). IR spectra were acquired on a FTIR and are reported in wave numbers $\left(\mathrm{cm}^{-1}\right) . R_{f}$ values refer to TLC on $0.25 \mathrm{~mm}$ silica gel plates $(60-\mathrm{F} 254)$. Flash chromatography was performed on silica gel $60(0.040-0.063 \mathrm{~mm})$. Melting points (mp) were determined on a capillary melting point apparatus and were uncorrected.

\section{Synthetic details for compounds 2-4:}

Compound 3: 8-methyl-8-azabicyclo [3.2.1] octan-3-yl 3,4dimethoxybenzoate (3)To a solution of commercial endo-tropine $1 \mathrm{~g}$ $7.08 \mathrm{mmol}, 1 \mathrm{eq}$ ) in dry DCM were added 2,3-dimethoxybenzoyl chloride (1.562 g, $7.78 \mathrm{mmol}, 1.1 \mathrm{eq})$, triethylamine (2.961 mL, $21.2 \mathrm{mmol}, 3 \mathrm{eq})$ and 4-dimethylaminopyridine $(0.951 \mathrm{~g}, 7.78 \mathrm{mmol}, 1.1 \mathrm{eq})$ under argon atmosphere. The reaction mixture was stirred at room temperature overnight before being cooled in $0^{\circ} \mathrm{C}$ and quenched with a saturated $\mathrm{NH}_{4} \mathrm{Cl}$ aqueous solution. The aqueous layer extracted with DCM (3 times). The combined organic layers were dried $\left(\mathrm{MgSO}_{4}\right)$, filtered and concentrated in vacuo. Purification by flash column chromatography on silica gel yielded the desired product ( $4.53 \mathrm{mmol})$ as a white solid.

Yield 64\%; white solid; TLC: $R_{f}=0.58$ (85:15 $\left.\mathrm{CH}_{2} \mathrm{Cl}_{2} / \mathrm{MeOH}\right)$, m.p.: 223 $224^{\circ} \mathrm{C}$. IR (neat): 2939, 2361, 1703, 1600, 1514, 1467, 1449, 1417, 1345 , $1272,1220,1175,1133,1025,877,764,632 \mathrm{~cm}^{-1} .{ }^{1} \mathrm{H}$ NMR $(300 \mathrm{MHz}$ $\left.\mathrm{CDCl}_{3}\right) \delta 7.56\left(\mathrm{dd}, 1 \mathrm{H}, J_{1}=8.4, J_{2}=1.9 \mathrm{~Hz}, \mathrm{H}_{6^{\prime}}\right), 7.51\left(\mathrm{~d}, 1 \mathrm{H}, J=1.9 \mathrm{~Hz}, \mathrm{H}_{2^{\prime}}\right.$ ) $\left.6.90\left(\mathrm{~d}, 1 \mathrm{H}, J=8.4 \mathrm{~Hz}, \mathrm{H}_{5}\right)^{\prime}\right), 5.37\left(\mathrm{~m}, 1 \mathrm{H}, \mathrm{H}_{3}\right), 3.94\left(\mathrm{~s}, 3 \mathrm{H}, \mathrm{H}_{7^{\prime}}\right), 3.92(\mathrm{~s}, 3 \mathrm{H}$, $\mathrm{H}_{8}$ ), 3.78 (br s, 2H, $\mathrm{H}_{1,5}$ ), 2.77 (s, 3H, $\left.\mathrm{H}_{9}\right), 2.55-2.25\left(\mathrm{~m}, 6 \mathrm{H}_{1} \mathrm{H}_{2,4,6 \alpha, 7 \alpha}\right.$ ), 2.20-2.05 (m, 2H, $\left.\mathrm{H}_{6 \beta, 7 \beta}\right) \cdot{ }^{13} \mathrm{C}$ NMR $\left(75 \mathrm{MHz}, \mathrm{CDCl}_{3}\right) \delta 165.19\left(\mathrm{C}_{8}\right), 153.53$ $\left(\mathrm{C}_{4^{\prime}}\right), 149.06\left(\mathrm{C}_{3^{\prime}}\right), 123.14\left(\mathrm{C}_{6^{\prime}}\right), 122.38\left(\mathrm{C}_{1^{\prime}}\right), 112.10\left(\mathrm{C}_{2^{\prime}}\right), 110.53\left(\mathrm{C}_{5^{\prime}}\right)$ $65.19\left(\mathrm{C}_{3}\right), 61.91\left(\mathrm{C}_{1,5}\right), 56.22\left(\mathrm{C}_{7^{\prime}}\right), 56.15\left(\mathrm{C}_{8^{\prime}}\right), 40.21\left(\mathrm{C}_{9}\right), 34.49\left(\mathrm{C}_{2,4}\right)$ $24.95\left(\mathrm{C}_{6,7}\right)$. MS $(\mathrm{m} / \mathrm{z}): 306.1705\left([\mathrm{M}+\mathrm{H}]^{+}\right)$.
Compound 4: 2,2,2-trichloroethyl3-((3,4-dimethoxybenzoyl)oxy)-8azabicyclo[3.2.1]octane-8-carboxylate (4) The Schlenk tube containing the $\mathrm{N}$-Meconvolvine $(0.1 \mathrm{~g}, 0.327 \mathrm{mmol}, 1 \mathrm{eq})$ was capped with a rubber septum, evacuated and backfilled with argon. This evacuation/backfill sequence was repeated one additional time. Dry toluene $(2 \mathrm{~mL})$ was added with 2,2,2-trichloroéthylechloroformate $(0.135 \mathrm{ml}, 0.982 \mathrm{mmol}, 3$ eq). The septum was replaced with a Teflon screwcap. The Schlenk tube was sealed, and the mixture was stirred at $140^{\circ} \mathrm{C}$ for 4 hours under microwave irradiations. The resulting suspension was cooled to room temperature and concentrated. The desired product is obtained as a white solid with a quantitative yield $(0.32 \mathrm{mmol})$.

White solid; TLC: $R_{f}=0.62$ (6:4 cyclohexane/EtOAc), m.p.: $135-136^{\circ} \mathrm{C}$, IR (neat): 2937, 2361, 1713, 1702, 1601, 1514, 1465,1414, 1313, 1289, 1270, 1248, 1221, 1178, 1102, 1078, 1023, 961, 942, 834, 762, 713, 632 $\mathrm{cm}^{-1} .{ }^{1} \mathrm{H}$ NMR (300 MHz, $\left.\mathrm{CDCl}_{3}\right) \delta 7.61\left(\mathrm{dd}, 1 \mathrm{H}, J_{1}=8.4, J_{2}=1.8 \mathrm{~Hz}, \mathrm{H}_{6}{ }^{\prime}\right.$ ), $7.52\left(\mathrm{~d}, 1 \mathrm{H}, J=1.8 \mathrm{~Hz}, \mathrm{H}_{2^{\prime}}\right), 6.89\left(\mathrm{~d}, 1 \mathrm{H}, J=8.4 \mathrm{~Hz}, \mathrm{H}_{5^{\prime}}\right), 5.33\left(\mathrm{~m}, 1 \mathrm{H}, \mathrm{H}_{3}\right)$, 4.84 (d, 1H, J = 12.0 Hz, H10a), 4.66 (d, 1H, J = $12.0 \mathrm{~Hz}, \mathrm{H}_{10 \mathrm{~b}}$ ), 4.41 (br s, $\left.2 \mathrm{H}, \mathrm{H}_{1,5}\right), 3.91$ (s, 3H, $\mathrm{H}_{7^{\prime}}$ ), 3.90 (s, 3H, $\mathrm{H}_{8^{\prime}}$ ), 2.37-2.03 (m, 6H, $\mathrm{H}_{2,4,6 \alpha, 7 \alpha}$ ), $1.93\left(\mathrm{~m}, 2 \mathrm{H}, \mathrm{H}_{6 \beta, 7 \beta}\right) .{ }^{13} \mathrm{C} \mathrm{NMR}\left(75 \mathrm{MHz}, \mathrm{CDCl}_{3}\right) \delta 165.45\left(\mathrm{C}_{8}\right), 153.15\left(\mathrm{C}_{9}\right)$, $151.40\left(\mathrm{C}_{4^{\prime}}\right), 148.81\left(\mathrm{C}_{3^{\prime}}\right), 123.24\left(\mathrm{C}_{6^{\prime}}\right), 122.96\left(\mathrm{C}_{1^{\prime}}\right), 111.98\left(\mathrm{C}_{2^{\prime}}\right), 110.45$ $\left(\mathrm{C}_{5^{\prime}}\right), 95.92\left(\mathrm{C}_{11}\right), 74.60\left(\mathrm{C}_{10}\right), 67.84\left(\mathrm{C}_{3}\right), 56.10\left(\mathrm{C}_{7^{\prime}}\right), 56.00\left(\mathrm{C}_{8^{\prime}}\right), 53.03$ $\left(\mathrm{C}_{1}\right), 52.90\left(\mathrm{C}_{5}\right), 36.38\left(\mathrm{C}_{2}\right), 35.61\left(\mathrm{C}_{4}\right), 28.55\left(\mathrm{C}_{6}\right), 27.70\left(\mathrm{C}_{7}\right) . \mathrm{MS}(\mathrm{m} / \mathrm{z})$ : $466.0526\left([\mathrm{M}+\mathrm{H}]^{+}\right)$.

Compound 2 (endo-convolvine): 8-azabicyclo[3.2.1]octan-3-yl 3,4dimethoxybenzoate (endo-convolvine 2)

The Schlenk tube was charged with the carbamate $(0.1 \mathrm{~g}, 0.214 \mathrm{mmol}, 1$ eq) and Zinc dust $(0.042 \mathrm{~g}, 0.642 \mathrm{mmol}, 3 \mathrm{eq})$ in acid acetic $(2 \mathrm{~mL})$. The tube was capped with a rubber septum, evacuated and backfilled with argon. This evacuation/backfill sequence was repeated one additional time. The septum was replaced with a Teflon screwcap. The Schlenk tube was sealed, and the mixture was stirred at $80^{\circ} \mathrm{C}$ during 2 hours. The resulting suspension was cooled to room temperature and quenched with of ammonia water concentrated and extracts three times with DCM. The aqueous layer extracted with DCM (3 times). The combined organic layers were dried $\left(\mathrm{MgSO}_{4}\right)$, filtered and concentrated in vacuo. Purification by flash column chromatography on silica gel yielded the desired product ( $0.192 \mathrm{mmol}$ ) as a white solid.

Yield 90\%; white solid; TLC: $R_{f}=0.36\left(8: 2 \mathrm{CH}_{2} \mathrm{Cl}_{2} / \mathrm{MeOH}\right)$, m.p. :101$102^{\circ} \mathrm{C}$,IR (neat): $2939,2837,1702,1601,1514,1465,1416,1357,1270$, $1224,1177,1132,1076,1023,875,849,765,629 \mathrm{~cm}^{-1} .{ }^{1} \mathrm{H}$ and ${ }^{13} \mathrm{C}$ $\operatorname{NMR}\left(75 \mathrm{MHz}, \mathrm{CDCl}_{3}\right) \delta 165.6,152.9,148.6,123.3,123.2,111.9,110.3$, 68.5, 55.9, $53.4(2 \mathrm{C}), 37.6,37.6,29.4(2 \mathrm{C}) . \mathrm{MS}(\mathrm{m} / \mathrm{z}): 292.1541\left([\mathrm{M}+\mathrm{H}]^{+}\right)$.

1. General procedure for Pd-Catalyzed coupling of convolvine with various aryls and heteroaryles:

A flame-dried resealable Schlenk tube was charged with $\mathrm{Pd}(\mathrm{OAc})_{2}(2 \mathrm{mg}$, $0.05 \mathrm{mmol}, 5 \mathrm{~mol} \%)$, Xphos ( $8 \mathrm{mg}, 0.10 \mathrm{mmol}, 10 \mathrm{~mol} \%)$, the solid reactant(s) (1.0 mmol of the bromoquinolin-2(1H)-one/aryl/heteroaryle bromides, $2 \mathrm{mmol}$ of the convolvine) and $t$-BuONa (33 $\mathrm{mg}, 2 \mathrm{mmol}$ ). The Schlenk tube was capped with a rubber septum, evacuated and backfilled with argon; this evacuation/backfill sequence was repeated one additional time. The liquid reactant 1,4-dioxane $(2 \mathrm{~mL})$ were added through the septum. The septum was replaced with a Teflon screwcap. The Schlenk tube was sealed, and the mixture was stirred at $100^{\circ} \mathrm{C}$ for 2 h. The resulting suspension was cooled to room temperature and filtred through Celite eluting with ethyl acetate, and the inorganic salts were removed. The filtrate was concentrated and purification of the residue by silica gel column chromatography gave the desired product.

Compound 5a: 8-(6-bromo-1-methyl-2-oxo-1,2-dihydroquinolin-3-yl)8azabicyclo [3.2.1] octan-3-yl 3,4-dimethoxybenzoate

Yield 97\% (0.97 mmol), yellow solid; m.p.: $146-147^{\circ} \mathrm{C}$; TLC: $R_{f} 0.38$ (Chloroforme/EtOAc: 95/5); IR (neat):2958, 1702, 1632, 1592, 1563, $1514,1463,1416,1379,1359,1342,1309,1289,1270,1248,1219$, $1177,1167,1132,1107,1084,1026,979,943,872,825,764,731,701$, $682,647,631 \mathrm{~cm}^{-1} .{ }^{1} \mathrm{H}$ NMR(400 MHz, Acetone- $\left.d_{6}\right) \delta 7.69\left(\mathrm{dd}, 1 \mathrm{H}, J_{1}=8.4\right.$, $\left.J_{2}=2.0 \mathrm{~Hz}, \mathrm{H}_{6}{ }^{\prime}\right), 7.58\left(\mathrm{~d}, 1 \mathrm{H}, J=1.9 \mathrm{~Hz}, \mathrm{H}_{8}{ }^{\prime \prime}\right), 7.53\left(\mathrm{dd}, 1 \mathrm{H}, J_{1}=7.7, J_{2}=1.3\right.$ $\left.\mathrm{Hz}, \mathrm{H}_{7^{\prime \prime}}\right), 7.40$ (d, $1 \mathrm{H}, J=8.3 \mathrm{~Hz}, \mathrm{H}_{2}$ ) , 7.35-7.29 (m, 1H, $\left.\mathrm{H}_{5}{ }^{\prime \prime}\right), 7.19-7.13$ (m, $\left.1 \mathrm{H}, \mathrm{H}_{3^{\prime \prime}}\right), 7.10\left(\mathrm{~d}, 1 \mathrm{H}, J=8.4 \mathrm{~Hz}, \mathrm{H}_{5^{\prime}}\right), 7.05\left(\mathrm{~s}, 1 \mathrm{H}, \mathrm{H}_{4^{\prime \prime}}\right), 5.26\left(\mathrm{~m}, 1 \mathrm{H}, \mathrm{H}_{3}\right)$, 
4.84 (br s, $\left.2 \mathrm{H}, \mathrm{H}_{1,5}\right), 3.91$ (s, $3 \mathrm{H}, \mathrm{H}_{7^{\prime}}$ ), 3.89 (s, $3 \mathrm{H}, \mathrm{H}_{8}$ ), 3.73 (s, $3 \mathrm{H}, \mathrm{H}_{9^{\prime \prime}}$ ), 2.40-2.34 (m, 2H, $\mathrm{H}_{2 \alpha, 4 \alpha}$ ), $2.32-2.27$ (m, 2H, $\left.\mathrm{H}_{2 \beta, 4 \beta}\right), 2.15-2.11$ (m, $2 \mathrm{H}$ $\left.\mathrm{H}_{6 \alpha, 7 \alpha}\right), 1.84$ (d, $\left.2 \mathrm{H}, J=14.4 \mathrm{~Hz}, \mathrm{H}_{6 \beta}, 7 \mathrm{\beta}\right) .{ }^{13} \mathrm{C}$ NMR(400 MHz,Acetone- $\left.d_{6}\right)$ $\delta 165.89\left(\mathrm{C}_{8}\right), \quad 159.55\left(\mathrm{C}_{2^{\prime \prime}}\right), \quad 154.45\left(\mathrm{C}_{4^{\prime}}\right), \quad 150.06\left(\mathrm{C}_{3^{\prime}}\right)^{\prime}, \quad 146.66\left(\mathrm{C}_{1^{\prime \prime}}\right)$, 137.94( $\left.\mathrm{C}_{8^{\prime \prime}} \mathrm{a}\right), \quad 136.19\left(\mathrm{C}_{4^{\prime \prime} \mathrm{a}}\right), \quad 127.07\left(\mathrm{C}_{5^{\prime \prime}}\right), \quad 126.56\left(\mathrm{C}_{7^{\prime \prime}}\right), \quad 124.00\left(\mathrm{C}_{6^{\prime \prime}}\right)$, $123.07\left(\mathrm{C}_{1^{\prime}}\right), \quad 122.79\left(\mathrm{C}_{6^{\prime}}\right), \quad 114.71\left(\mathrm{C}_{8^{\prime \prime}}\right), \quad 114.46\left(\mathrm{C}_{2^{\prime}}\right), \quad 113.00\left(\mathrm{C}_{5^{\prime}}\right)$ 111.92( $\left(\mathrm{C}_{4^{\prime \prime}}\right), \quad 69.24\left(\mathrm{C}_{3}\right), 56.23\left(\mathrm{C}_{7^{\prime}}\right), 56.12\left(\mathrm{C}_{8^{\prime}}\right), 55.01\left(\mathrm{C}_{1,5}\right), 34.70\left(\mathrm{C}_{2,4}\right)$, 31.58( $\left(\mathrm{C}_{9^{\prime \prime}}\right), 28.26\left(\mathrm{C}_{6,7}\right) . \mathrm{MS}(\mathrm{m} / \mathrm{z}): 449.1559\left([\mathrm{M}+\mathrm{H}]^{+}\right)$.

Compound 5b: 8-(4-fluorophenyl)-8-azabicyclo [3.2.1] octan-3-yl 3,4dimethoxybenzoate

Yield 70\% (0.70 mmol),, yellow solid; m.p.: $180-181^{\circ} \mathrm{C}$; TLC: $R_{f} 0.44$ (Cyclohexane/EtOAc: 8/2); IR (neat): 3050, 2956, 2937, 2915, 2838, $1702,1601,1589,1517,1504,1465,1442,1416,1377,1358,1345$ 1328, 1291, 1270, 1249, 1227, 1211, 1175, 1132, 1107, 1085, 1033, 987, 948, 929, 880, 871, 819, 802, 764, 734, 707, $632 \mathrm{~cm}^{-1} .{ }^{1} \mathrm{H}$ NMR(300 MHz, $\left.\mathrm{CDCl}_{3}\right) \delta 7.68\left(\mathrm{dd}, 1 \mathrm{H}, J_{1}=8.4, J_{2}=1.6 \mathrm{~Hz}, \mathrm{H}_{6}{ }^{\prime}\right), 7.58\left(\mathrm{~d}, 1 \mathrm{H}, J=1.6 \mathrm{~Hz}, \mathrm{H}_{2}{ }^{\prime}\right.$ )

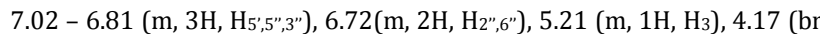
s, $\left.2 \mathrm{H}, \mathrm{H}_{1,5}\right), 3.95\left(\mathrm{~s}, 3 \mathrm{H}, \mathrm{H}_{7^{\prime}}\right), 3.94\left(\mathrm{~s}, 3 \mathrm{H}, \mathrm{H}_{8^{\prime}}\right), 2.46-2.09\left(\mathrm{~m}, 6 \mathrm{H}, \mathrm{H}_{2,4,6 \alpha, 7 \alpha}\right.$ ), $1.76\left(\mathrm{~d}, 2 \mathrm{H}, J=15.2 \mathrm{~Hz}, \mathrm{H}_{6 \beta}, 7 \beta\right) .{ }^{13} \mathrm{C} \mathrm{NMR}\left(75 \mathrm{MHz}_{2} \mathrm{CDCl}_{3}\right) \delta 165.73\left(\mathrm{C}_{8}\right)$, $157.15\left(\mathrm{C}_{4^{\prime \prime}}\right), 153.17\left(\mathrm{C}_{4^{\prime}}\right), 148.90\left(\mathrm{C}_{3^{\prime}}\right), 142.92\left(\mathrm{C}_{1^{\prime \prime}}\right), 123.37\left(\mathrm{C}_{6^{\prime}}\right)$ $116.29\left(\mathrm{C}_{1^{\prime}}\right), 116.05\left(\mathrm{C}_{3^{\prime \prime}, 5^{\prime \prime}}\right), 116.00\left(\mathrm{C}_{2^{\prime \prime}, 6^{\prime \prime}}\right), 112.09\left(\mathrm{C}_{2^{\prime}}\right), 110.54\left(\mathrm{C}_{5^{\prime}}\right), 68.73$ $\left(\mathrm{C}_{3}\right), 56.17\left(\mathrm{C}_{7^{\prime}}\right), 56.08\left(\mathrm{C}_{8^{\prime}}\right), 53.63\left(\mathrm{C}_{1,5}\right), 31.41\left(\mathrm{C}_{2,4}\right), 28.32\left(\mathrm{C}_{6,7}\right) . \mathrm{MS}$ $(\mathrm{m} / \mathrm{z}): 386.1770\left([\mathrm{M}+\mathrm{H}]^{+}\right)$.

Compound 5c: 8-(4-(trifluoromethyl)phenyl)-8-azabicyclo[3.2.1]octan-3yl 3,4-dimethoxybenzoate

Yield 57\% (0.57 mmol), white solid; m.p. :179-180 ${ }^{\circ} \mathrm{C}$, TLC: $R_{f} 0.50$ (Cyclohexane/EtOAc: 7/3); IR (neat): 2956, 2937, 2915, 2838, 1702, $1601,1589,1517,1504,1465,1442,1416,1377,1358,1345,1328$ $1291,1270,1249,1227,1211,1175,1132,1107,1085,1033,987,948$ $929,880,871,819,802,764,734,707,632 \mathrm{~cm}^{-1}$. ${ }^{1} \mathrm{H}$ NMR $(300 \mathrm{MHz}$ $\left.\mathrm{CDCl}_{3}\right) \delta 7.69\left(\mathrm{dd}, 1 \mathrm{H}, J_{1}=8.4, J_{2}=1.6 \mathrm{~Hz}, \mathrm{H}_{6^{\prime}}{ }^{\prime}\right), 7.58\left(\mathrm{~d}, 1 \mathrm{H}, J=1.6 \mathrm{~Hz}, \mathrm{H}_{2^{\prime}}\right.$ ),

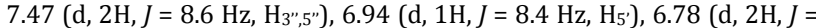
$8.6 \mathrm{~Hz}, \mathrm{H}_{2 \prime}, 6^{\prime \prime}$ ), 5.22 (m, 1H, H3), 4.30 (br s, $2 \mathrm{H}, \mathrm{H}_{1,5}$ ), 3.95 (s, 3H, $\mathrm{H}_{7^{\prime}}$ ), 3.94

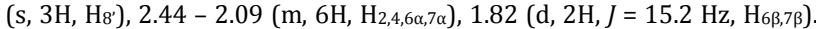
${ }^{13} \mathrm{C} \quad \mathrm{NMR} \quad\left(75 \mathrm{MHz}, \mathrm{CDCl}_{3}\right) \delta \quad 165.69\left(\mathrm{C}_{8}\right), \quad 153.24\left(\mathrm{C}_{1^{\prime \prime}}\right), \quad 148.94\left(\mathrm{C}_{4^{\prime}}\right)$, $148.57\left(\mathrm{C}_{3^{\prime}}\right), \quad 127.07\left(\mathrm{C}_{3^{\prime \prime}}\right), \quad 127.02\left(\mathrm{C}_{5^{\prime \prime}}\right), \quad 123.38\left(\mathrm{C}_{6^{\prime}}\right), \quad 123.25\left(\mathrm{C}_{1^{\prime}}\right)$, $118.69\left(\mathrm{C}_{4^{\prime \prime}}\right), \quad 118.26\left(\mathrm{C}_{7^{\prime \prime}}\right), \quad 114.05\left(\mathrm{C}_{2^{\prime \prime}, 6^{\prime \prime}}\right), \quad 112.11\left(\mathrm{C}_{2^{\prime}}\right), \quad 110.56\left(\mathrm{C}_{5^{\prime}}\right)$ 68.59 $\left(\mathrm{C}_{3}\right), 56.20\left(\mathrm{C}_{7^{\prime}}\right), 56.10\left(\mathrm{C}_{8^{\prime}}\right), 53.07\left(\mathrm{C}_{1,5}\right), 31.94\left(\mathrm{C}_{2,4}\right), 28.30\left(\mathrm{C}_{6,7}\right)$. MS $(\mathrm{m} / \mathrm{z}): 436.1736\left([\mathrm{M}+\mathrm{H}]^{+}\right)$.

Compound 5d: 8-(4-chlorophenyl)-8-azabicyclo[3.2.1]octan-3-yl 3,4dimethoxybenzoate

Yield 58\% (0.58 mmol),, white solid; m.p.: $170-171^{\circ} \mathrm{C}$; TLC: $R_{f} 0.61$ (Cyclohexane/EtOAc: 7/3); IR (neat):2957, 2935, 2916, 2837, 1710, $1703,1596,1514,1494,1464,1443,1416,1379,1358,1345,1329$, 1296, 1270, 1248, 1225, 1216, 1176, 1133, 1098, 1082, 1032, 987, 948, 930, 881, 871, 814, 765, 736, 700,682, $632 \mathrm{~cm}^{-1} .{ }^{1} \mathrm{H}$ NMR(300 MHz $\left.\mathrm{CDCl}_{3}\right) \delta 7.68\left(\mathrm{dd}, 1 \mathrm{H}, J_{1}=8.4, J_{2}=1.8 \mathrm{~Hz}, \mathrm{H}_{6^{\prime}}\right), 7.58\left(\mathrm{~d}, 1 \mathrm{H}, J=1.8 \mathrm{~Hz}, \mathrm{H}_{2^{\prime}}\right.$ ),

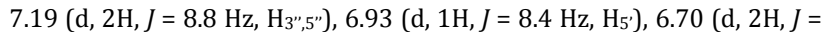
$\left.8.9 \mathrm{~Hz}, \mathrm{H}_{2 \prime \prime}, 6^{\prime \prime}\right), 5.20$ (m, 1H, $\mathrm{H}_{3}$ ), 4.20 (br s, $\left.2 \mathrm{H}, \mathrm{H}_{1,5}\right), 3.95$ (s, 3H, $\mathrm{H}_{7^{\prime}}$ ), 3.94 (s, 3H, $\left.\mathrm{H}_{8^{\prime}}\right), 2.42-2.06\left(\mathrm{~m}, 6 \mathrm{H}, \mathrm{H}_{2,4,6 \alpha, 7 \alpha}\right), 1.76\left(\mathrm{~d}, 2 \mathrm{H}, J=15.2 \mathrm{~Hz}, \mathrm{H}_{6 \beta, 7 \beta}\right.$ ). ${ }^{13} \mathrm{C}$ NMR $\left(75 \mathrm{MHz}, \mathrm{CDCl}_{3}\right) \delta 165.71\left(\mathrm{C}_{8}\right), 153.19\left(\mathrm{C}_{4^{\prime}}\right), \quad 148.91\left(\mathrm{C}_{3^{\prime}}\right)$ $144.95\left(\mathrm{C}_{1^{\prime \prime}}\right), \quad 129.55\left(\mathrm{C}_{3^{\prime \prime}, 5^{\prime \prime}}\right), \quad 123.37\left(\mathrm{C}_{6^{\prime}}\right), \quad 123.33\left(\mathrm{C}_{4^{\prime \prime}}\right), \quad 121.88\left(\mathrm{C}_{1^{\prime}}\right)$ $116.21\left(\mathrm{C}_{2^{\prime \prime}, 6^{\prime \prime}}\right), 112.09\left(\mathrm{C}_{2^{\prime}}\right), 110.55\left(\mathrm{C}_{5^{\prime}}\right), 68.70\left(\mathrm{C}_{3}\right), 56.18\left(\mathrm{C}_{7^{\prime}}\right), 56.09\left(\mathrm{C}_{8^{\prime}}\right)$, 53.26 $\left(\mathrm{C}_{1,5}\right), 31.46\left(\mathrm{C}_{2,4}\right), 28.33\left(\mathrm{C}_{6,7}\right)$. MS (m/z): $402.1468\left([\mathrm{M}+\mathrm{H}]^{+}\right)$.

Compound 5e: 8-(4-nitrophenyl)-8-azabicyclo[3.2.1]octan-3-yl 3,4dimethoxybenzoate

Yield 58\% (0.58 mmol),, yellow solid; m.p.: 202-203우 TLC: $R_{f} 0.59$ (Cyclohexane/EtOAc: 7/3); IR (neat): 2960, 2917, 2850, 2214, 1710, $1705,1605,1514,1494,1465,1446,1417,1383,1348,1306,1290$ $1272,1249,1223,1178,1168,1134,1107,1086,1031,988,945,930$ 883, 871, 822, 766, 736, 699, $632 \mathrm{~cm}^{-1} .{ }^{1} \mathrm{H}$ NMR(300 MHz, $\left.\mathrm{CDCl}_{3}\right) \delta 8.15$ $\left(\mathrm{d}, 2 \mathrm{H}, J=9.2 \mathrm{~Hz}, \mathrm{H}_{3}{ }^{\prime \prime}, 5^{\prime \prime}\right), 7.67\left(\mathrm{dd}, 1 \mathrm{H}, J_{1}=8.4, J_{2}=1.8 \mathrm{~Hz}, \mathrm{H}_{6^{\prime}}\right), 7.57(\mathrm{~d}, 1 \mathrm{H}, J$ $=1.8 \mathrm{~Hz}, \mathrm{H}_{2^{\prime}}$ ), $6.94\left(\mathrm{~d}, 1 \mathrm{H}, J=8.4 \mathrm{~Hz}, \mathrm{H}_{5^{\prime}}\right.$ ), 6.70 (d, $\left.2 \mathrm{H}, J=9.2 \mathrm{~Hz}, \mathrm{H}_{2 \prime \prime}, 6^{\prime \prime}\right)$, $5.26\left(\mathrm{~m}, 1 \mathrm{H}, \mathrm{H}_{3}\right), 4.39$ (br s, $\left.2 \mathrm{H}, \mathrm{H}_{1,5}\right), 3.96$ (s, 3H, $\left.\mathrm{H}_{7^{\prime}}\right), 3.95$ (s, 3H, $\mathrm{H}_{8^{\prime}}$ ) 2.47-2.10 (m, 6H, $\mathrm{H}_{2,4,6 \alpha, 7 \alpha)} 1.92$ (d, 2H, J = $\left.15.2 \mathrm{~Hz}, \mathrm{H}_{6 \beta, 7 \beta}\right) .{ }^{13} \mathrm{C}$ NMR(75 $\left.\mathrm{MHz}, \quad \mathrm{CDCl}_{3}\right) \delta \quad 165.63\left(\mathrm{C}_{8}\right), \quad 153.38\left(\mathrm{C}_{1^{\prime}}\right), \quad 150.67\left(\mathrm{C}_{4^{\prime}}\right), \quad 149.01\left(\mathrm{C}_{3^{\prime}}\right)$ $137.51\left(\mathrm{C}_{4^{\prime \prime}}\right), \quad 126.86\left(\mathrm{C}_{3^{\prime \prime}}\right), \quad 126.77\left(\mathrm{C}_{5^{\prime \prime}}\right), 123.40\left(\mathrm{C}_{6^{\prime}}\right), \quad 123.04\left(\mathrm{C}_{1^{\prime}}\right)$,
112.82( $\left.\mathrm{C}_{2^{\prime \prime}, 6^{\prime \prime}}\right), 112.14\left(\mathrm{C}_{2^{\prime}}\right), 110.60\left(\mathrm{C}_{5^{\prime}}\right), 68.17\left(\mathrm{C}_{3}\right), 56.24\left(\mathrm{C}_{7^{\prime}}\right), 56.15\left(\mathrm{C}_{8^{\prime}}\right)$, 53.61 $\left(\mathrm{C}_{1,5}\right), 33.25\left(\mathrm{C}_{2,4}\right), 28.20\left(\mathrm{C}_{6,7}\right)$. MS (APCI positive, $\mathrm{m} / \mathrm{z}$ ): 413.1705 $\left([\mathrm{M}+\mathrm{H}]^{+}\right)$.

Compound 5f: 8-(4-cyanophenyl)-8-azabicyclo[3.2.1]octan-3-yl 3,4dimethoxybenzoate

Yield 76\% (0.76 mmol),, white solid; m.p.: $206-207^{\circ} \mathrm{C}$; TLC: $R_{f} 0.40$ (Cyclohexane/EtOAc: 7/3); IR (neat):3055, 2961, 2927, 2854, 1704, $1605,1588,1513,1464,1444,1420,1383,1350,1292,1266,1223$, $1179,1168,1134,1104,1080,1032,986,943,870,842,822,796,766$, 733, 702, 664, $630 \mathrm{~cm}^{-1} .{ }^{1} \mathrm{H} \mathrm{NMR}\left(300 \mathrm{MHz}, \mathrm{CDCl}_{3}\right) \delta 7.67$ (dd, $1 \mathrm{H}, J_{1}=8.4$, $J_{2}=1.6 \mathrm{~Hz}, \mathrm{H}_{6^{\prime}}$ ), 7.57 (d, $\left.1 \mathrm{H}, J=1.6 \mathrm{~Hz}, \mathrm{H}_{2}{ }^{\prime}\right), 7.49$ (d, $\left.2 \mathrm{H}, J=8.8 \mathrm{~Hz}, \mathrm{H}_{3^{\prime \prime}}, 5^{\prime \prime}\right)$, $6.93\left(\mathrm{~d}, 1 \mathrm{H}, J=8.4 \mathrm{~Hz}, \mathrm{H}_{5^{\prime}}\right.$ ), 6.74 (d, 2H, $\left.J=8.8 \mathrm{~Hz}, \mathrm{H}_{2 \prime}{ }^{\prime \prime} 6^{\prime \prime}\right), 5.23\left(\mathrm{~m}, 1 \mathrm{H}, \mathrm{H}_{3}\right)$, 4.31 (br s, $\left.2 \mathrm{H}, \mathrm{H}_{1,5}\right), 3.95$ (s, 3H, $\left.\mathrm{H}_{7^{\prime}}\right), 3.94\left(\mathrm{~s}, 3 \mathrm{H}, \mathrm{H}_{8^{\prime}}\right), 2.42-2.09(\mathrm{~m}, 6 \mathrm{H}$, $\left.\mathrm{H}_{2,4,6 \alpha, 7 \alpha}\right), 1.86\left(\mathrm{~d}, 2 \mathrm{H}, J=15.3 \mathrm{~Hz}, \mathrm{H}_{6 \beta}, 7 \beta\right) .{ }^{13} \mathrm{C} \mathrm{NMR}\left(75 \mathrm{MHz}, \mathrm{CDCl}_{3}\right) \delta$ $165.65\left(\mathrm{C}_{8}\right), \quad 153.31\left(\mathrm{C}_{1^{\prime}}\right), \quad 148.93\left(\mathrm{C}_{4^{\prime}, 3^{\prime}}\right), \quad 134.13\left(\mathrm{C}_{3^{\prime \prime}, 5^{\prime \prime}}\right), \quad 123.38\left(\mathrm{C}_{6^{\prime}}\right)$, $123.11\left(\mathrm{C}_{1^{\prime}}\right), \quad 120.49\left(\mathrm{C}_{7^{\prime \prime}}\right), \quad 114.21\left(\mathrm{C}_{2^{\prime}}\right), \quad 112.11\left(\mathrm{C}_{5^{\prime}}\right), \quad 110.58\left(\mathrm{C}_{2^{\prime \prime}, 6^{\prime \prime}}\right)$, 98.47( $\left(\mathrm{C}_{4^{\prime \prime}}\right), \quad 68.33\left(\mathrm{C}_{3}\right), \quad 56.22\left(\mathrm{C}_{7^{\prime}}\right), \quad 56.12\left(\mathrm{C}_{8^{\prime}}\right), \quad 53.11\left(\mathrm{C}_{1,5}\right), \quad 32.52\left(\mathrm{C}_{2,4}\right)$, 28.23 $\left(\mathrm{C}_{6,7}\right)$. MS (m/z): $393.1815\left([\mathrm{M}+\mathrm{H}]^{+}\right)$.

Compound 5g: 8-(2-cyanophenyl)-8-azabicyclo[3.2.1]octan-3-yl 3,4dimethoxybenzoate

Yield 30\% (0.30 mmol),, white solid; m.p. :138-139 ${ }^{\circ} \mathrm{C}$; TLC: $R_{f} 0.35$ (Cyclohexane/EtOAc: 7/3); IR (neat): 2959, 2214, 1703, 1598, 1514, 1486, 1465, 1446, 1417, 1380, 1358, 1290,1269, 1247, 1222, 1177, 1133, 1107, 1072, 1029, 935, 882, 814, 765, 733, 708, $631 \mathrm{~cm}^{-1}{ }^{1} \mathrm{H}$ $\mathrm{NMR}\left(300 \mathrm{MHz}, \mathrm{CDCl}_{3}\right) \delta 7.67\left(\mathrm{dd}, 1 \mathrm{H}, J_{1}=8.4, J_{2}=1.6 \mathrm{~Hz}, \mathrm{H}_{6^{\prime}}\right), 7.57(\mathrm{~d}, 1 \mathrm{H}, J$ = $\left.1.6 \mathrm{~Hz}, \mathrm{H}_{2^{\prime}}\right), 7.52\left(\mathrm{~d}, 1 \mathrm{H}, J=7.7 \mathrm{~Hz}, \mathrm{H}_{3^{\prime \prime}}\right), 7.39$ (t, $1 \mathrm{H}, J=7.2 \mathrm{~Hz}, \mathrm{H}_{5^{\prime \prime}}$ ), 6.98-6.77 (m, 3H, $\left.\mathrm{H}_{5}^{\prime}, 4^{\prime \prime}, 6^{\prime \prime}\right), 5.35$ (m, 1H, $\mathrm{H}_{3}$ ), 4.43 (br s, 2H, $\mathrm{H}_{1,5}$ ), 3.95 (s, $3 \mathrm{H}, \mathrm{H}_{7^{\prime}}$ ), 3.94 (s, 3H, $\mathrm{H}_{8^{\prime}}$ ), 2.51-2.38 (m, $\left.2 \mathrm{H}, \mathrm{H}_{2 \alpha, 4 \alpha}\right), 2.28-2.24(\mathrm{~m}, 2 \mathrm{H}$, $\left.\mathrm{H}_{2 \beta, 4 \beta}\right), 2.12-2.06\left(\mathrm{~m}, 2 \mathrm{H}, \mathrm{H}_{6 \alpha, 7 \alpha}\right), 2.00\left(\mathrm{~d}, 2 \mathrm{H}, J=16.4 \mathrm{~Hz}, \mathrm{H}_{6 \beta, 7 \beta}\right) .{ }^{13} \mathrm{C} \mathrm{NMR}$ $\left(75 \mathrm{MHz}, \mathrm{CDCl}_{3}\right) \delta 165.68\left(\mathrm{C}_{8}\right), 153.18\left(\mathrm{C}_{4^{\prime}}\right), 152.14\left(\mathrm{C}_{1^{\prime \prime}}\right), 148.90\left(\mathrm{C}_{3^{\prime}}\right)$, $135.29\left(\mathrm{C}_{5^{\prime \prime}}\right), \quad 133.84\left(\mathrm{C}_{3^{\prime \prime}}\right), \quad 123.37\left(\mathrm{C}_{6^{\prime}}\right), \quad 123.29\left(\mathrm{C}_{1^{\prime}}\right), \quad 119.79\left(\mathrm{C}_{7^{\prime \prime}}\right)$, 119.37( $\left.\mathrm{C}_{4^{\prime \prime}}\right), 116.61\left(\mathrm{C}_{6^{\prime \prime}}\right), 112.13\left(\mathrm{C}_{2^{\prime}}\right), 110.54\left(\mathrm{C}_{5^{\prime}}\right), 101.59\left(\mathrm{C}_{2^{\prime \prime}}\right), 68.23\left(\mathrm{C}_{3}\right)$, 57.28 $\left(\mathrm{C}_{1,5}\right), \quad 56.18\left(\mathrm{C}_{7^{\prime}}\right), 56.10\left(\mathrm{C}_{8^{\prime}}\right), 36.15\left(\mathrm{C}_{2,4}\right), 27.73\left(\mathrm{C}_{6,7}\right)$. MS $(\mathrm{m} / \mathrm{z})$ : $393.1811\left([\mathrm{M}+\mathrm{H}]^{+}\right)$.

Compound 5h: 8-(3-cyanophenyl)-8-azabicyclo[3.2.1]octan-3-yl 3,4dimethoxybenzoate

Yield 44\% (0.44 mmol), white solid; m.p.:149-150 ${ }^{\circ} \mathrm{C}$; TLC: $R f 0.35$ (Cyclohexane/EtOAc: 7/3); IR (neat): 2958, 2838, 2359, 2227, 1705, 1598, 1571, 1515, 1492, 1466, 1438, 1417, 1379, 1338, 1305, 1290, $1272,1218,1177,1133,1109,1083,1033,982,942,873,765,733,682$, $632 \mathrm{~cm}^{-1} .{ }^{1} \mathrm{H}$ NMR $\left(300 \mathrm{MHz}, \mathrm{CDCl}_{3}\right) \delta 7.68\left(\mathrm{dd}, 1 \mathrm{H}, J_{1}=8.4, J_{2}=1.6 \mathrm{~Hz}, \mathrm{H}_{6}{ }^{\prime}\right)$, $7.57\left(\mathrm{~d}, 1 \mathrm{H}, J=1.6 \mathrm{~Hz}, \mathrm{H}_{2}\right), 7.30\left(\mathrm{t}, 1 \mathrm{H}, J=7.9 \mathrm{~Hz}, \mathrm{H}_{5}{ }^{\prime \prime}\right), 7.06-6.82(\mathrm{~m}, 4 \mathrm{H}$,

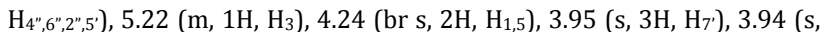
$\left.3 \mathrm{H}, \mathrm{H}_{8^{\prime}}\right), 2.43-2.11\left(\mathrm{~m}, 6 \mathrm{H}, \mathrm{H}_{2,4,6 \alpha, 7 \alpha}\right), 1.82\left(\mathrm{~d}, 2 \mathrm{H}, J=15.3 \mathrm{~Hz}, \mathrm{H}_{6 \beta}, 7 \beta\right) .{ }^{13} \mathrm{C}$ NMR $\left(75 \mathrm{MHz}, \mathrm{CDCl}_{3}\right) \delta 165.66\left(\mathrm{C}_{8}\right), 153.25\left(\mathrm{C}_{4^{\prime}}\right), 148.93\left(\mathrm{C}_{1^{\prime \prime}}\right), 146.47\left(\mathrm{C}_{3^{\prime}}\right)$, $130.48\left(\mathrm{C}_{5^{\prime \prime}}\right), \quad 123.38\left(\mathrm{C}_{6^{\prime}}\right), \quad 123.17\left(\mathrm{C}_{1^{\prime}}\right), \quad 120.37\left(\mathrm{C}_{4^{\prime \prime}}\right), \quad 119.63\left(\mathrm{C}_{7^{\prime \prime}}\right)$, 119.02( $\left(\mathrm{C}_{6^{\prime \prime}}\right), 117.64\left(\mathrm{C}_{2^{\prime \prime}}\right), 113.55\left(\mathrm{C}_{3^{\prime \prime}}\right), 112.09\left(\mathrm{C}_{2^{\prime}}\right), 110.56\left(\mathrm{C}_{5^{\prime}}\right), 68.40\left(\mathrm{C}_{3}\right)$, $56.20\left(\mathrm{C}_{7^{\prime}}\right), 56.10\left(\mathrm{C}_{8^{\prime}}\right), 53.16\left(\mathrm{C}_{1,5}\right), 31.74\left(\mathrm{C}_{2,4}\right), 28.27\left(\mathrm{C}_{6,7}\right)$. MS $(\mathrm{m} / \mathrm{z})$ : $393.1811\left([\mathrm{M}+\mathrm{H}]^{+}\right)$.

Compound 5i: 8-(4-methoxyphenyl)-8-azabicyclo[3.2.1]octan-3-yl 3,4dimethoxybenzoate

Yield 88\% (0.88 mmol),, white solid; m.p. :136-137 C; TLC: $R_{f} 0.54$ (Cyclohexane/EtOAc: 7/3); IR (neat): 3051, 2960,2933, 2854, 1710, $1707,1603,1510,1465,1445,1418,1381,1360,1291,1266,1242$, $1224,1215,1179,1132,1108,1086,1034,1004,930,879,853,821$, $791,767,733,702,632 \mathrm{~cm}^{-1} .{ }^{1} \mathrm{H}$ NMR $\left(300 \mathrm{MHz}, \mathrm{CDCl}_{3}\right) \delta 7.69\left(\mathrm{dd}, 1 \mathrm{H}, \mathrm{J}_{1}=\right.$ 8.4, $\left.J_{2}=1.5 \mathrm{~Hz}, \mathrm{H}_{6^{\prime}}\right), 7.59\left(\mathrm{~d}, 1 \mathrm{H}, J=1.5 \mathrm{~Hz}, \mathrm{H}_{2^{\prime}}\right), 6.93(\mathrm{~d}, 1 \mathrm{H}, J=8.4 \mathrm{~Hz}$, $\mathrm{H}_{5^{\prime}}$ ), 6.86 (d, 2H, J = 8.9 Hz, $\left.\mathrm{H}_{2 "}{ }^{\prime \prime} 6^{\prime \prime}\right), 6.76$ (d, 2H, J = 9.0 Hz, H $\left.3^{\prime \prime}, 5^{\prime \prime}\right), 5.22$ (m, $1 \mathrm{H}, \mathrm{H}_{3}$ ), 4.18 (br s, $2 \mathrm{H}, \mathrm{H}_{1,5}$ ), 3.95 (s, 3H, $\mathrm{H}_{7^{\prime}}$ ), 3.94 (s, 3H, $\mathrm{H}_{8^{\prime}}$ ), 3.77 (s, $3 \mathrm{H}$, $\left.\mathrm{H}_{7^{\prime \prime}}\right), 2.48-2.09\left(\mathrm{~m}, 6 \mathrm{H}, \mathrm{H}_{2,4,6 \alpha, 7 \alpha}\right), 1.75\left(\mathrm{~d}, 2 \mathrm{H}, J=15.1 \mathrm{~Hz}, \mathrm{H}_{6 \beta}, 7 \beta\right) .{ }^{13} \mathrm{C}$ NMR $\left(75 \mathrm{MHz}, \mathrm{CDCl}_{3}\right) \delta 165.71\left(\mathrm{C}_{8}\right), 153.09\left(\mathrm{C}_{4^{\prime}}\right), 151.89\left(\mathrm{C}_{4^{\prime \prime}}\right), 148.85\left(\mathrm{C}_{3^{\prime}}\right)$, $140.74\left(\mathrm{C}_{1 "}\right), \quad 123.41\left(\mathrm{C}_{6^{\prime}}\right), \quad 123.33\left(\mathrm{C}_{1^{\prime}}\right), \quad 116.35\left(\mathrm{C}_{2^{\prime \prime}, 6^{\prime \prime}}\right), \quad 115.24\left(\mathrm{C}_{3^{\prime \prime}, 5^{\prime \prime}}\right)$, $112.06\left(\mathrm{C}_{2^{\prime}}\right), 110.51\left(\mathrm{C}_{5^{\prime}}\right), 68.86\left(\mathrm{C}_{3}\right), 56.13\left(\mathrm{C}_{7^{\prime}}\right), 56.03\left(\mathrm{C}_{8^{\prime}}\right), 55.77\left(\mathrm{C}_{7^{\prime \prime}}\right)$, 53.71 $\left(\mathrm{C}_{1,5}\right), 31.50\left(\mathrm{C}_{2,4}\right), 28.25\left(\mathrm{C}_{6,7}\right), 27.00 . \mathrm{MS}(\mathrm{m} / \mathrm{z}): 398.1968\left([\mathrm{M}+\mathrm{H}]^{+}\right)$.

Compound 5j: 8-(4-methoxyphenyl)-8-azabicyclo[3.2.1]octan-3-yl 3,4dimethoxybenzoate 
Yield 96\% (0.96 mmol),, white solid; m.p. :129-130 ${ }^{\circ}$; TLC: $R_{f} 0.42$ (Cyclohexane/EtOAc: 7/3); IR (neat): 2956, 2836, 2255, 1704, 1601, 1516, 1498, 1466, 1453, 1417, 1378, 1356, 1291, 1271, 1221, 1177, $1130,1108,1069,1030,933,910,881,813,766,728,648,631 \mathrm{~cm}^{-1} .{ }^{1} \mathrm{H}$ NMR (300 MHz, CDCl 3$) \delta 7.69\left(\mathrm{dd}, 1 \mathrm{H}, J_{1}=8.4, J_{2}=1.8 \mathrm{~Hz}, \mathrm{H}_{6}{ }^{\prime}\right), 7.59(\mathrm{~d}, 1 \mathrm{H}$ $J=1.8 \mathrm{~Hz}, \mathrm{H}_{2^{\prime}}$ ), 6.92 (d, $1 \mathrm{H}, J=8.4 \mathrm{~Hz}, \mathrm{H}_{5^{\prime}}$ ), 6.86-6.83 (m, 4H, $\mathrm{H}_{3 \prime \prime}, 4^{\prime \prime}, 5^{\prime \prime}, 6^{\prime \prime}$ ), $5.34\left(\mathrm{~m}, 1 \mathrm{H}, \mathrm{H}_{3}\right), 4.21$ (br s, $\left.2 \mathrm{H}, \mathrm{H}_{1,5}\right), 3.94$ (s, 6H, $\mathrm{H}_{7^{\prime}, 8^{\prime}}$ ), 3.86 (s, $3 \mathrm{H}_{1} \mathrm{H}_{7^{\prime \prime}}$ ), 2.52-2.35 (m, $\left.2 \mathrm{H}, \mathrm{H}_{2 \alpha, 4 \alpha}\right), 2.28-2.02\left(\mathrm{~m}, 4 \mathrm{H}, \mathrm{H}_{2 \beta, 4 \beta, 6 \alpha, 7 \alpha)} 1.91(\mathrm{~d}, 2 \mathrm{H}, J=\right.$ $\left.14.8 \mathrm{~Hz}, \mathrm{H}_{6 \beta, 7 \beta}\right) .{ }^{13} \mathrm{C}$ NMR $\left(75 \mathrm{MHz}, \mathrm{CDCl}_{3}\right) \delta 165.75\left(\mathrm{C}_{8}\right), 153.01\left(\mathrm{C}_{4}\right)^{\prime}$, $151.22\left(\mathrm{C}_{2^{\prime \prime}}\right), \quad 148.80\left(\mathrm{C}_{3^{\prime}}\right), \quad 138.72\left(\mathrm{C}_{1^{\prime \prime}}\right), \quad 123.55\left(\mathrm{C}_{1^{\prime}}\right), \quad 123.35\left(\mathrm{C}_{6^{\prime}}\right)$, $121.14\left(\mathrm{C}_{4^{\prime \prime}}\right), \quad 120.92\left(\mathrm{C}_{3^{\prime \prime}}\right), \quad 116.85\left(\mathrm{C}_{6^{\prime \prime}}\right), \quad 112.09\left(\mathrm{C}_{2^{\prime}}\right), \quad 111.94\left(\mathrm{C}_{5^{\prime \prime}}\right)$ 110.49( $\left.\mathrm{C}_{5^{\prime}}\right), \quad 68.77\left(\mathrm{C}_{3}\right), 56.44\left(\mathrm{C}_{7^{\prime}}\right), 56.12\left(\mathrm{C}_{8^{\prime}}\right), 56.04\left(\mathrm{C}_{7^{\prime \prime}}\right), 55.61\left(\mathrm{C}_{1,5}\right)$, 36.36( $\left(\mathrm{C}_{2,4}\right), 27.65\left(\mathrm{C}_{6,7}\right)$. MS (m/z): $398.1968\left([\mathrm{M}+\mathrm{H}]^{+}\right)$.

Compound 5k: 8-(4-methoxyphenyl)-8-azabicyclo[3.2.1]octan-3-yl 3,4dimethoxybenzoate

Yield 57\% (0.57 mmol),, white solid; m.p. :123-124 ${ }^{\circ} \mathrm{C}$; TLC: $R_{f} 0.44$ (Cyclohexane/EtOAc: 7/3); IR (neat): 2958,2836, 2253, 1710, 1610, $1600,1573,1515,1494,1465,1417,1359,1290,1271,1222,1178$, $1164,1133,1108,1081,1032,983,941,909,872,765,727,688,648$ $\mathrm{cm}^{-1} .{ }^{1} \mathrm{H} \mathrm{NMR}\left(300 \mathrm{MHz}, \mathrm{CDCl}_{3}\right) \delta 7.69\left(\mathrm{dd}, 1 \mathrm{H}, J_{1}=8.4, J_{2}=1.8 \mathrm{~Hz}, \mathrm{H}_{6}\right)$, $7.58\left(\mathrm{~d}, 1 \mathrm{H}, J=1.8 \mathrm{~Hz}, \mathrm{H}_{2}\right), 7.17\left(\mathrm{t}, 1 \mathrm{H}, J=8.1 \mathrm{~Hz}, \mathrm{H}_{5}^{\prime \prime}\right), 6.93(\mathrm{~d}, 1 \mathrm{H}, J=8.4$ $\left.\left.\mathrm{Hz}, \mathrm{H}_{5}\right)^{\prime}\right), 6.42$ (dd, 1H, $\left.J_{1}=8.1, J_{2}=1.7 \mathrm{~Hz}, \mathrm{H}_{6}^{\prime \prime}\right), 6.38-6.19\left(\mathrm{~m}, 2 \mathrm{H}, \mathrm{H}_{2}{ }^{\prime \prime} 4^{\prime \prime}\right.$, $5.22\left(\mathrm{~m}, 1 \mathrm{H}, \mathrm{H}_{3}\right), 4.23$ (br s, $\left.2 \mathrm{H}, \mathrm{H}_{1,5}\right), 3.94$ (s, $3 \mathrm{H}, \mathrm{H}_{7}$ ), 3.93 (s, $3 \mathrm{H}, \mathrm{H}_{8}$ ) 3.80 (s, 3H, $\left.\mathrm{H}_{7^{\prime \prime}}\right), 2.44-2.05\left(\mathrm{~m}, 6 \mathrm{H}, \mathrm{H}_{2,4,6 \alpha, 7 \alpha}\right), 1.76(\mathrm{~d}, 2 \mathrm{H}, J=15.2 \mathrm{~Hz}$ $\left.\mathrm{H}_{6 \beta, 7 \beta}\right) .{ }^{13} \mathrm{C}$ NMR $\left.\left(75 \mathrm{MHz}, \mathrm{CDCl}_{3}\right) \delta 165.78\left(\mathrm{C}_{8}\right), 161.21\left(\mathrm{C}_{3^{\prime \prime}}\right), 153.16\left(\mathrm{C}_{4}\right)^{\prime}\right)$ 148.91( $\left.\mathrm{C}_{3^{\prime}}\right), \quad 147.65\left(\mathrm{C}_{1^{\prime \prime}}\right), \quad 130.49\left(\mathrm{C}_{5^{\prime \prime}}\right), \quad 123.39\left(\mathrm{C}_{6^{\prime}}\right), \quad 112.12\left(\mathrm{C}_{2^{\prime}}\right)$ $111.19\left(\mathrm{C}_{1^{\prime}}\right), 110.56\left(\mathrm{C}_{5^{\prime}}\right), 108.15\left(\mathrm{C}_{4^{\prime \prime}}\right), 101.99\left(\mathrm{C}_{6^{\prime \prime}}\right), 101.72\left(\mathrm{C}_{2^{\prime \prime}}\right), 68.98\left(\mathrm{C}_{3}\right)$, 56.21( $\left.\mathrm{C}_{7^{\prime}}\right), 56.11\left(\mathrm{C}_{8^{\prime}}\right), 55.32\left(\mathrm{C}_{7^{\prime \prime}}\right), 53.16\left(\mathrm{C}_{1,5}\right), 31.68\left(\mathrm{C}_{2,4}\right), 28.36\left(\mathrm{C}_{6,7}\right)$. MS $(\mathrm{m} / \mathrm{z}): 398.1968\left([\mathrm{M}+\mathrm{H}]^{+}\right)$.

Compound 5l: 8-phenyl-8-azabicyclo[3.2.1]octan-3-yl 3,4dimethoxybenzoate

Yield 68\% (0.68 mmol),, white solid; m.p.:183-184 $\mathrm{C}$; TLC: $R_{f} 0.48$ (Cyclohexane/EtOAc: 7/3); IR (neat): 2360, 2341, 2330, 1704, 1597, $1511,1460,1418,1387,1321,1303,1271,1226,1193,1174,1140$ 1114, 1086, 1029, 872, 831, 766, 752, $724 \mathrm{~cm}^{-1} .{ }^{1} \mathrm{H}$ NMR $(300 \mathrm{MHz}$, $\left.\mathrm{CDCl}_{3}\right) \delta 7.70\left(\mathrm{dd}, 1 \mathrm{H}, J_{1}=8.4, J_{2}=1.8 \mathrm{~Hz}, \mathrm{H}_{6}{ }^{\prime}\right), 7.59\left(\mathrm{~d}, 1 \mathrm{H}, J=1.8 \mathrm{~Hz}, \mathrm{H}_{2^{\prime}}\right.$ ), 7.29 (s, 1H, $\left.\mathrm{H}_{3^{\prime \prime}}\right), 7.23$ (s, 1H, $\left.\mathrm{H}_{5}{ }^{\prime \prime}\right), 6.94\left(\mathrm{~d}, 1 \mathrm{H}, J=8.4 \mathrm{~Hz}, \mathrm{H}_{5^{\prime}}\right), 6.80$ (d, 2H, $\left.=8.5 \mathrm{~Hz}, \mathrm{H}_{2 "}, 6^{\prime \prime}\right), 6.72\left(\mathrm{t}, 1 \mathrm{H}, J=7.3 \mathrm{~Hz}, \mathrm{H}_{4 "}\right), 5.22\left(\mathrm{~m}, 1 \mathrm{H}, \mathrm{H}_{3}\right), 4.27$ (br s, $\left.2 \mathrm{H}, \mathrm{H}_{1,5}\right), 3.96\left(\mathrm{~s}, 3 \mathrm{H}, \mathrm{H}_{7^{\prime}}\right), 3.95$ (s, 3H, $\mathrm{H}_{8^{\prime}}$ ), $2.48-2.04\left(\mathrm{~m}, 6 \mathrm{H}, \mathrm{H}_{2,4,6 \alpha, 7 \alpha}\right.$ ), $1.77\left(\mathrm{~d}, 2 \mathrm{H}, J=15.1 \mathrm{~Hz}, \mathrm{H}_{6 \beta, 7 \beta}\right) .{ }^{13} \mathrm{C}$ NMR $\left(75 \mathrm{MHz}, \mathrm{CDCl}_{3}\right) \delta 165.74\left(\mathrm{C}_{8}\right)$, 153.13( $\left.\mathrm{C}_{4^{\prime}}\right), \quad 148.88\left(\mathrm{C}_{3^{\prime}}\right), \quad 146.30\left(\mathrm{C}_{1^{\prime \prime}}\right), \quad 129.72\left(\mathrm{C}_{3^{\prime \prime}, 5^{\prime \prime}}\right)$, $123.42\left(\mathrm{C}_{6^{\prime}}\right), 123.36\left(\mathrm{C}_{1^{\prime}}\right), \quad 117.25\left(\mathrm{C}_{4^{\prime \prime}}\right), \quad 115.07\left(\mathrm{C}_{2^{\prime \prime}, 6^{\prime \prime}}\right), \quad 112.08\left(\mathrm{C}_{2^{\prime}}\right)$, 110.53( $\left.\mathrm{C}_{5^{\prime}}\right), 68.96\left(\mathrm{C}_{3}\right), 56.17\left(\mathrm{C}_{7^{\prime}}\right), 56.07\left(\mathrm{C}_{8^{\prime}}\right), 52.94\left(\mathrm{C}_{1,5}\right), 31.53\left(\mathrm{C}_{2,4}\right)$, 28.33( $\left.\mathrm{C}_{6,7}\right) \cdot \mathrm{MS}(\mathrm{m} / \mathrm{z}): 368.1870\left([\mathrm{M}+\mathrm{H}]^{+}\right)$.

Compound 5m: 8-(1-methyl-1H-indol-5-yl)-8-azabicyclo[3.2.1]octan-3-yl 3,4-dimethoxybenzoate

Yield 70\% (0.70 mmol),, white solid; m.p. : $188-189^{\circ} \mathrm{C} ; R_{f} 0.31$ (Cyclohexane/EtOAc: 7/3); IR (neat): 2957, 1702, 1624, 1601, 1570 , $1514,1491,1464,1448,1418,1379,1353,1291,1270,1247,1218$, 1177, 1163, 1132, 1107, 1083, 1033, 941, 877, 822, 765, 728, 648, 631 $\mathrm{cm}^{-1} .{ }^{1} \mathrm{H}$ NMR (300 MHz, $\left.\mathrm{CDCl}_{3}\right) \delta 7.76\left(\mathrm{dd}, 1 \mathrm{H}, J_{1}=8.4, J_{2}=1.8 \mathrm{~Hz}, \mathrm{H}_{6^{\prime}}\right)$, $7.65\left(\mathrm{~d}, 1 \mathrm{H}, J=1.6 \mathrm{~Hz}, \mathrm{H}_{2^{\prime}}\right), 7.30\left(\mathrm{~d}, 1 \mathrm{H}, J=4.1 \mathrm{~Hz}, \mathrm{H}_{5^{\prime}}\right), 7.06(\mathrm{~d}, 1 \mathrm{H}, J=1.7$ $\left.\mathrm{Hz}, \mathrm{H}_{4}{ }^{\prime \prime}\right), 7.02$ (d, 1H, $\left.J=3.0 \mathrm{~Hz}, \mathrm{H}_{3^{\prime \prime}}\right), 6.98$ (d, 1H, $\left.J=8.4 \mathrm{~Hz}, \mathrm{H}_{6^{\prime \prime}}\right), 6.93$ (dd, $\left.1 \mathrm{H}, J_{1}=8.9, J_{2}=1.9 \mathrm{~Hz}, \mathrm{H}_{7 "}\right), 6.39\left(\mathrm{~d}, 1 \mathrm{H}, J=2.9 \mathrm{~Hz}, \mathrm{H}_{2^{\prime \prime}}\right), 5.25\left(\mathrm{~m}, 1 \mathrm{H}, \mathrm{H}_{3}\right)$

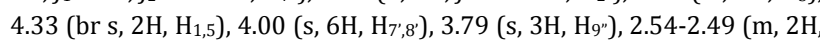

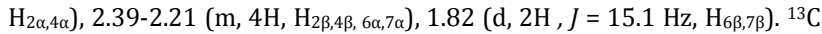
NMR $\left(75 \mathrm{MHz}, \mathrm{CDCl}_{3}\right) \delta 165.80\left(\mathrm{C}_{8}\right), 153.06\left(\mathrm{C}_{4^{\prime}}\right), 148.84\left(\mathrm{C}_{3^{\prime}}\right), 140.52\left(\mathrm{C}_{1^{\prime \prime}}\right)$ $131.15\left(\mathrm{C}_{4^{\prime \prime} \mathrm{a}}\right), \quad 129.69\left(\mathrm{C}_{4^{\prime \prime} \mathrm{b}}\right), \quad 129.16\left(\mathrm{C}_{6^{\prime \prime}}\right), \quad 123.56\left(\mathrm{C}_{1^{\prime}}\right), 123.38\left(\mathrm{C}_{6^{\prime}}\right)$, 112.61( $\left.\mathrm{C}_{2^{\prime}}\right), 112.09\left(\mathrm{C}_{5^{\prime}}\right), 110.53\left(\mathrm{C}_{3^{\prime \prime}}\right), 110.20\left(\mathrm{C}_{2^{\prime \prime}}\right), 105.92\left(\mathrm{C}_{8^{\prime \prime}}\right), 99.96\left(\mathrm{C}_{7^{\prime \prime}}\right)$, 69.17( $\left.\mathrm{C}_{3}\right), \quad 56.17\left(\mathrm{C}_{7^{\prime}}\right), \quad 56.07\left(\mathrm{C}_{8^{\prime}}\right), \quad 54.17\left(\mathrm{C}_{1,5}\right), \quad 32.97\left(\mathrm{C}_{9^{\prime \prime}}\right), 31.82\left(\mathrm{C}_{2,4}\right)$, 28.28( $\left(\mathrm{C}_{6,7}\right)$. MS (m/z): $421.2122\left([\mathrm{M}+\mathrm{H}]^{+}\right)$.

Compound 5n: 8-(pyridin-3-yl)-8-azabicyclo[3.2.1]octan-3-yl 3,4dimethoxybenzoate

Yield 95\% (0.95 mmol),, white solid; m.p. :140-141 ${ }^{\circ} \mathrm{C}$; TLC: $R_{f} 0.45$ ( $\mathrm{CH}_{2} \mathrm{Cl}_{2}$ /EtOAc: 4/6); IR (neat): 2957, 2838, 2360, 1703, 1601, 1580, $1515,1487,1465,1418,1378,1349,1302,1290,1272,1248,1218$, $1177,1133,1108,1087,1032,986,948,930,823,798,765,730,707$
632, $614 \mathrm{~cm}^{-1} .{ }^{1} \mathrm{H}$ NMR (300 MHz, $\left.\mathrm{CDCl}_{3}\right) \delta 8.19$ (br s, $\left.1 \mathrm{H}, \mathrm{H}_{2 \prime \prime}\right), 7.98$ (br s, $\left.1 \mathrm{H}, \mathrm{H}_{4^{\prime \prime}}\right), 7.67\left(\mathrm{dd}, 1 \mathrm{H}, J_{1}=8.4, J_{2}=1.4 \mathrm{~Hz}, \mathrm{H}_{6^{\prime}}{ }^{\prime}\right), 7.56\left(\mathrm{~d}, 1 \mathrm{H}, J=1.4 \mathrm{~Hz}, \mathrm{H}_{2^{\prime}}\right.$ ), 7.12 - $7.16\left(\mathrm{~m}, 1 \mathrm{H}, \mathrm{H}_{5}{ }^{\prime \prime}\right), 7.04$ (d, 1H, $\left.J=8.4 \mathrm{~Hz}, \mathrm{H}_{6}{ }^{\prime \prime}\right), 6.92$ (d, $1 \mathrm{H}, J=8.4$ $\mathrm{Hz}, \mathrm{H}_{5^{\prime}}$ ), $5.20\left(\mathrm{~m}, 1 \mathrm{H}, \mathrm{H}_{3}\right), 4.26$ (br s, $\left.2 \mathrm{H}, \mathrm{H}_{1,5}\right), 3.93$ (s, 3H, $\left.\mathrm{H}_{7^{\prime}}\right), 3.92(\mathrm{~s}, 3 \mathrm{H}$,

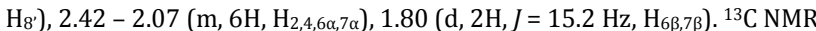
$\left(75 \mathrm{MHz}_{1} \mathrm{CDCl}_{3}\right) \delta \quad 165.62\left(\mathrm{C}_{8}\right), 153.18\left(\mathrm{C}_{4^{\prime}}\right), 148.87\left(\mathrm{C}_{3^{\prime}}\right), 142.29\left(\mathrm{C}_{1^{\prime \prime}}\right)$, $138.48\left(\mathrm{C}_{4}^{\prime \prime}\right), \quad 137.57\left(\mathrm{C}_{2^{\prime \prime}}\right), \quad 124.06\left(\mathrm{C}_{1^{\prime}}\right), \quad 123.33\left(\mathrm{C}_{6^{\prime}}\right), \quad 123.19\left(\mathrm{C}_{5^{\prime \prime}}\right)$, $121.49\left(\mathrm{C}_{6^{\prime \prime}}\right), 112.05\left(\mathrm{C}_{2^{\prime}}\right), 110.52\left(\mathrm{C}_{5^{\prime}}\right), 68.44\left(\mathrm{C}_{3}\right), 56.15\left(\mathrm{C}_{7^{\prime}}\right), 56.05\left(\mathrm{C}_{8^{\prime}}\right)$, 52.83( $\left(\mathrm{C}_{1,5}\right), 31.58\left(\mathrm{C}_{2,4}\right), 28.18\left(\mathrm{C}_{6,7}\right) . \mathbf{M S}(\mathrm{m} / \mathrm{z}): 369.1811\left([\mathrm{M}+\mathrm{H}]^{+}\right)$.

Compound 5o: $8-(6-((1 R, 3 R, 5 S)-3-((3,4-$ dimethoxybenzoyl $)$ oxy $)-8$ azabicyclo[3.2.1]octan-8-yl)-1-

methyl-2-oxo-1,2-dihydroquinolin-3-yl)-8-azabicyclo[3.2.1]octan-3-yl3,4dimethoxy benzoate

Yield 43\% (0.43 mmol),, white solid; m.p.: $118-119^{\circ} \mathrm{C}$; TLC: $R_{f} 0.51$ ( $\mathrm{CH}_{2} \mathrm{Cl}_{2}$ /EtOAc: 9/1); IR (neat): 2958, 2917, 2851, 1703, 1633, 1604, $1589,1514,1465,1439,1417,1380,1346,1290,1271,1249,1222$, $1178,1168,1133,1107,1085,1031,987,944,931,880,872,821,798$, 765, 734, 701, $632 \mathrm{~cm}^{-1} .{ }^{1} \mathrm{H}$ NMR (300 MHz, Acetone- $\left.d_{6}\right) \delta 7.69(\mathrm{dd}, 2 \mathrm{H}$, $\left.J_{1}=8.4, J_{2}=1.9 \mathrm{~Hz}, \mathrm{H}_{6^{\prime}}, 6^{\prime \prime \prime}\right), 7.58$ (br s, $\left.2 \mathrm{H}, \mathrm{H}_{2^{\prime}}, 2^{\prime \prime \prime}\right), 7.31$ (d, $1 \mathrm{H}, J=8.8 \mathrm{~Hz}$,

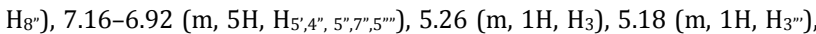
4.83 (br s, $2 \mathrm{H}, \mathrm{H}_{1,5}$ ), 4.35 (br s, $2 \mathrm{H}, \mathrm{H}_{\left.1^{\prime \prime}, 5^{\prime \prime}\right)}$ ), 3.91 (s, 6H, $\mathrm{H}_{7^{\prime}, 8^{\prime}}$ ), 3.89 (s, 6H, $\mathrm{H}_{\left.7^{\prime \prime \prime}, 8^{\prime \prime \prime}\right),} 3.69$ (s, 3H, $\left.\mathrm{H}_{9 "}\right), 2.80$ (br s, $\left.2 \mathrm{H}, \mathrm{H}_{2 \alpha, 4 \alpha}\right), 2.44-2.22(\mathrm{~m}, 6 \mathrm{H}$, $\mathrm{H}_{2 \beta, 4 \beta, 6,7)}$, 1.87-1.76 (m, 8H, $\left.\mathrm{H}_{2 "}, 4^{\prime \prime}, 6^{\prime \prime}, 7^{\prime \prime}\right) .{ }^{13} \mathrm{C}$ NMR (75 MHz, Acetone- $\left.d_{6}\right) \delta$ 165.87 ( $\left.\mathrm{C}_{8,8^{\prime \prime \prime}}\right), 154.46\left(\mathrm{C}_{2^{\prime \prime}}\right), 150.05\left(\mathrm{C}_{4^{\prime}, 4^{\prime \prime \prime}}\right), 138.16\left(\mathrm{C}_{3^{\prime}, 3^{\prime \prime \prime}}\right), 136.66\left(\mathrm{C}_{6^{\prime \prime}}\right)$,

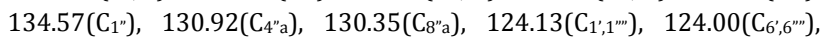
$115.60\left(\mathrm{C}_{2,2^{\prime \prime \prime}}\right), \quad 114.72\left(\mathrm{C}_{7 "}\right), \quad 112.98\left(\mathrm{C}_{5^{\prime}, 5^{\prime \prime \prime}}\right), \quad 111.90\left(\mathrm{C}_{4 ", 5}{ }^{\prime \prime}\right), \quad 69.27\left(\mathrm{C}_{3,3^{\prime \prime \prime}}\right)$, 56.24 $\left(\mathrm{C}_{7^{\prime}, 8^{\prime}}\right), \quad 56.12\left(\mathrm{C}_{\left.7^{\prime \prime \prime}, 8^{\prime \prime \prime}\right)}\right), 55.00\left(\mathrm{C}_{1,5,1^{\prime \prime \prime}, 5^{\prime \prime \prime}}\right), 34.68\left(\mathrm{C}_{2,4,2^{\prime \prime \prime}, 4^{\prime \prime \prime}}\right), 32.26\left(\mathrm{C}_{9^{\prime \prime}}\right)$, $28.27\left(\mathrm{C}_{\left.6,7,6^{\prime \prime}, 7^{\prime \prime}\right) .}\right) \mathrm{MS}(\mathrm{m} / \mathrm{z}): 738.3414\left([\mathrm{M}+\mathrm{H}]^{+}\right)$.

Protocol for Cell Culture and Proliferation Assay: Cancer cell lines were obtained from the American type Culture Collection (Rockville, MD) and were cultured according to the supplier's instructions. Briefly, HCT116 cells were grown in RPMI 1640 containing 10\% FCS and 1\% glutamine. All cell lines were maintained at $37{ }^{\circ} \mathrm{C}$ in a humidified atmosphere containing $5 \% \quad \mathrm{CO}_{2}$. Cell viability was assessed using Promega CellTiter-Blue TM reagent according to the manufacturer's instructions. Cells were seeded in 96-well plates $(5 \times 103$ cells/well) containing $50 \mu \mathrm{L}$ growth medium. After $24 \mathrm{~h}$ of culture, the cells were supplemented with $50 \mu \mathrm{L}$ of the tested compound dissolved in DMSO (less than $0.1 \%$ in each preparation). After $72 \mathrm{~h}$ of incubation, $20 \mu \mathrm{L}$ of resazurin was added for $2 \mathrm{~h}$ before recording fluorescence $(\lambda \mathrm{ex}=560$ $\mathrm{nm}, \lambda \mathrm{em}=590 \mathrm{~nm}$ ) using a Victor microtiter plate fluorimeter (PerkinElmer, USA). The $\mathrm{IC}_{50}$ corresponds to the concentration of the tested compound that caused a decrease of $50 \%$ in fluorescence of drug treated cells compared with untreated cells. Experiments were performed in triplicate.

\section{Funding Information}

Click here to insert sources of funding, grant numbers, etc. Do not repeat the same in the acknowledgment.

\section{Acknowledgment}

Authors acknowledge support of this project by CNRS, University Paris Sud and by La Ligue Nationale Contre le Cancer throughout an Equipe Labellisée 2014 grant. The authors are also grateful to Prof. Fethia Harzallah-Skhiri, High Institute of Biotechnology of Monastir, Tunisia, for botanical identification of the plant material and to the Ministry of Higher Education and Scientific Research of Tunisia for financial support.Our laboratory (BioCIS UMR 8076) is a member of the laboratory of excellence LERMIT supported by a grant from ANR (ANR10-LABX-33).

\section{Supporting Information}

Supporting information for this article $(1 \mathrm{H}, 13 \mathrm{C}$, COSY, HSQC, HMBC and NOESY NMR spectra) is available online at

\section{References}


(1) Lounasmaa, M.; Tamminen T.; The Alkaloids (Ed: G.A. Cordell), Academic Press, New York, 1993, 44, 1.

(2) (a) Kukula-Koch, W.A.; Widelski, J. in Pharmacognosy, Pharmacognosy Fundamentals, Applications and Strategies 2017, 163; (b) Grynkiewicz, G.; Gadzikowska, M.Pharmacol.Rep. 2008, 60, 439.

(3) Grynkiewicz, G.; Gadzikowska, M. Acta Pol Pharm - Drug Res. 2008, 60, 439 .

(4) Bussenius, J.; Blazey, C. M.; Aay, N.; Anand, N. K.; Arcalas, A.; Baik, T.; Bowles, O. J.; Buhr, C. A.; Costanzo, S.; Curtis, J. K.; DeFina, S. C.; Dubenko, L.; Heuer, T. S.; Huang, P.; Jaeger, C.; Joshi, A.; Kennedy, A. R.; Kim, A. I.; Lara, K.; Lee, J.; Li, J.; Lougheed, J. C.; Ma, S.; Malek, S.; Manalo, J. C.; Martini, J. F.; McGrath, G.; Nicoll, M.; Nuss, J. M.; Pack, M.; Peto, C. J.; Tsang, T. H.; Wang, L.; Womble, S. W.; Yakes, M.; Zhang, W.; Rice, K. D. Bioorg. Med. Chem. Lett. 2012, 22, 5396.

(5) Audisio, D.; Messaoudi, S.; Cegielkowski, L.; Peyrat, J.-F.; Brion, J.D.; Methy-Gonnot, D.; Radanyi, C.;Renoir, J.-M.; Alami, M. Chem. Med. Chem. 2011, 6, 804.

(6) Audisio, D.; Messaoudi, S.; Peyrat, J.-F.; Brion, J.-D.; Alami, M. J. Org. Chem. 2011, 76, 4995-5005.

(7) Yagudaev, M. R., Aripova, S. F. Khim. Prir. Soedin. 1986, 1, 80.
(8) Sharova, E. G.; Aripova, S. F.; Yu Yunusov S. Khim. Prir. Soedin.1980, 672.

(9) (a) Aripova, S. F.; Yu Yunusov, S. Khim. Prir.Soedin. 1979, 527; (b) Aripova, S. F.; Sharova, E. G.; Yu Yunusov, S.; Dzhabbarov, A. Khim. Prir. Soedin. 1983, 245.

(10) Maksay, G. Nemes, P. Bíró, T. J. Med. Chem. 2004, 47, 6384

(11) Crystallographic data (excluding structure factors) for the structure of $\mathrm{N}$-methylconvolvine 3 have been deposited with the Cambridge Crystallographic Data Centre as supplementary publication number CCDC 1535462. Copies of the data can be obtained, free of charge, on application to CCDC, 12 Union Road, Cambridge CB2 1EZ, UK (fax: +44(0)-1223-336033 or e-mail: deposit@ccdc.cam.ac.uk).

(12) (a) Kraiss, G.; Nádor. K. Tetrahedron Lett. 1971,1, 57 (b) Wallace, J. L.; Kidd, M. R.; Cauthen, S. E.; Woodyard, J. D. J. Pharm. Sci. 1980, 69, 1357; (b) Do Pham, D. D. Kelso, G. F. Yang, Y. Hearn, M. T. W. Green Chem. 2012, 14, 1189.

(13) Messaoudi, S.; Audisio, D.; Brion, J.-D.; Alami, M. Tetrahedron, 2007,63, 10202 . 